\title{
Electronic energy transfer in biomacromolecules
}

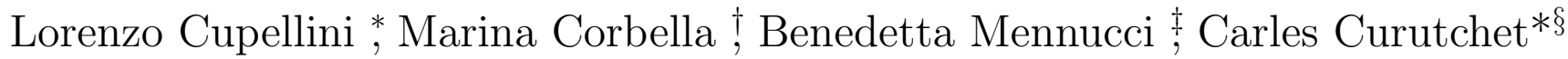

Article Type:

Advanced Review

\begin{abstract}
Electronic energy transfer is widely used as a molecular ruler to interrogate the structure of biomacromolecules, and performs a key task in photosynthesis by transferring collected energy through specialized pigment-protein complexes. Förster theory, introduced over 70 years ago, allows linking transfer rates to simple structural and spectroscopic properties of the energy-transferring molecules. In biosystems, however, significant deviations from Förster behavior often arise due to breakdown of the ideal dipole approximation, dielectric screening effects due to the biological environment, or departure from the weak-coupling regime. In this review, we provide a concise overview of advances in simulations of energy transfer in biomacromolecules that allow overcoming the main limitations of Förster theory. We first discuss advances in quantum chemical methods to compute electronic couplings, their extension to multiscale formulations to include screening effects, and strategies to treat the interplay between coupling fluctuations and energy transfer dynamics. We then examine the spectral overlap term, and how this quantity can be estimated from simulations of the spectral density of exciton-phonon coupling. Finally, we discuss rate theories that can describe energy transfers in situations where strong coupling leads to delocalized excitions, a common situation found in closely packed multichromophoric systems like photosynthetic complexes and nucleic acids.
\end{abstract}

* ORCID 0000-0003-0848-2908, Dipartimento di Chimica e Chimica Industriale, University of Pisa, Italy. Email: lorenzo.cupellini@for.unipi.it

†ORCID 0000-0001-9209-68X, Departament de Farmàcia i Tecnologia Farmacèutica i Fisicoquímica and Institut de Química Teòrica i Computacional (IQTC-UB), Facultat de Farmàcia i Ciències de l'Alimentació, Universitat de Barcelona, Spain. Email: marinacorbella@ub.edu

†ORCID 0000-0002-4394-0129, Dipartimento di Chimica e Chimica Industriale, University of Pisa, Italy. Email: benedetta.mennucci@unipi.it

$\S$ ORCID 0000-0002-0070-1208, Departament de Farmàcia i Tecnologia Farmacèutica i Fisicoquímica and Institut de Química Teòrica i Computacional (IQTC-UB), Facultat de Farmàcia i Ciències de l'Alimentació, Universitat de Barcelona, Spain. Email: carles.curutchet@ub.edu 


\section{INTRODUCTION}

Electronic energy transfer (EET) is a ubiquitous process in materials and life sciences, which describes the non-radiative transfer of electronic excitation energy from a previously sensitized donor (D) molecule to a proximate acceptor (A), up to distances of $10 \mathrm{~nm}$. ${ }^{1}$ The foundations of our present understanding of EET were settled about 70 years ago by Theodor Förster, ${ }^{2,3}$ who introduced an elegant theory based on purely spectroscopic observables. This theory had an immense impact in a variety of fields. In particular, in chemistry and biology, the term FRET (Förster resonance energy transfer) is indistinctively used to refer either to the photophysical process or to the fluorescence-based technique that uses energy transfer as a spectroscopic ruler to measure distances, given the strong dependence of EET efficiency on D/A mutual distance and orientation. ${ }^{4}$ A properly calibrated FRET experiment in principle allows one to achieve an accurate estimate of the D/A distance, although a recurring problem is the determination of their relative orientation. For such a reason, FRET data often have to be complemented by other techniques. ${ }^{5}$ Molecular simulations have emerged as an ideal complement to FRET due to its ability to explore the complex energy landscapes of biomolecules and provide structural models with atomic detail, including the relative D/A orientation, that can be compared to the information derived from fluorescence experiments.

Since the diffusion of single-molecule detection techniques in the 1990s, the FRET technique has been key toward the development of a dynamic structural biology able to overcome the limitations of static structures as those typically inferred from x-ray crystallography, nu-

clear magnetic resonance (NMR) or cryo-electron microscopy (cryo-EM). Indeed, nowadays, single-molecule FRET (smFRET) experiments allow investigating transient conformations of biomolecules and the associated interconversion dynamics, even in vivo, with great detail. The smFRET technique is used, for instance, to investigate the conformations of intrinsicallydisordered proteins or the mechanisms of protein and RNA folding. ${ }^{5,6}$

Energy transfer plays also a pivotal role in the chain of events that, in photosynthesis, transport the energy captured by antenna complexes to reaction centers. ${ }^{7,8}$ Other applications of EET processes in biosystems are in the mechanisms of photoreceptors ${ }^{9}$ or DNA photodamage, given that energy migration can occur along the double helix after photon 
absorption or sensitization. ${ }^{10}$ Also in these cases, molecular simulations are widely used to relate the structure of photosynthetic pigment-protein complexes to their light-harvesting properties, or to rationalize the energy transfer mechanisms. ${ }^{8}$

In spite of the fundamental role played by the original Förster theory, extensive studies on bridged molecular dyads, photosynthetic systems, and organic and nanostructured materials, have identified a number of cases where energy transfer rates significantly deviate from Förster's predictions. ${ }^{11}$ For example, the important interplay between the timescales of EET and those of the conformational dynamics is a fundamental issue that is not accounted for in standard applications of Förster theory. Moreover, in systems like DNA or photosynthetic complexes, characterized by short interchromophoric separations, different assumptions underlying Förster theory need to be overcome.

In this review, we aim at providing a succinct overview of the issues that a computational chemist should consider when modelling EET in biomacromolecules. Basically, which theoretical method should be adopted in order to compute excited states and electronic couplings, which strategies can be used to account for structural dynamics on EET rates, and in which situations one needs to go beyond the weak-coupling assumption underlying Förster theory. Our aim is to provide a unified view of the simulation strategies that are currently pursued by a variety of laboratories in modelling biosystems as diverse as nucleic acids, protein-ligand complexes, photosynthetic antenna proteins or fluorophore-tagged biomolecules.

We start by introducing Förster formulation of energy transfer and the ingredients needed to estimate the rate: the electronic coupling and the spectral overlap factor. We continue by describing quantum chemical methods aimed at predicting the electronic coupling going beyond Förster's main approximations: the point-dipole approximation, the assumption of a simple environment dielectric screening factor, or the neglect of short-range contributions, which become important at contact distances or in transfers involving forbidden transitions. In parallel, we also discuss the interplay between structural dynamics and energy transfer timescales and suitable strategies to post-process electronic coupling data sets generated from Molecular Dynamics (MD) trajectories. We then discuss issues involved in the determination of the spectral overlap factor and the spectral lineshapes, and its relation to dynamic and static disorder. Finally, in the last section we describe situations where the weak-coupling 
assumption underlying Förter theory breaks down, for example, when a strong coupling leads to delocalized exciton states, which play an important role in multichromophoric aggregates.

\section{FÖRSTER EXPRESSION OF THE EET RATE}

Förster theory describes the rate of energy transfer between a pair of weakly-coupled D and A molecules based on the Fermi Golden rule, Eq. (1): ${ }^{2,12}$

$$
k_{\mathrm{EET}}=\frac{2 \pi}{\hbar}|V|^{2} \mathrm{FCWD}
$$

where $V$ indicates the electronic coupling between D/A and FCWD is the Franck-Condon factor weighted density of states.

Within the original Förster formulation, $V$ is approximated as a dipole-dipole interaction screened by an environmental factor determined by the inverse of the squared refractive index $\left(1 / n^{2}\right)$ :

$$
\begin{gathered}
V \approx \frac{1}{n^{2}} V_{d d} \\
V_{d d}=\frac{\kappa \mu_{D} \mu_{A}}{R^{3}} \\
\kappa=\hat{\mu}_{D} \cdot \hat{\mu}_{A}-3\left(\hat{\mu}_{D} \cdot \hat{R}\right)\left(\hat{\mu}_{A} \cdot \hat{R}\right)
\end{gathered}
$$

where $\mu_{D}$ and $\mu_{A}$ indicate the magnitude of the $\mathrm{D} / \mathrm{A}$ transition dipole moments, $R$ the separation between the two dipoles, and the orientation factor $\kappa$ is given in terms of unit vectors, indicated by the hat notation.

To proceed further, the FCWD can be rewritten in terms of the spectral overlap factor obtained from the area-normalized donor emission and acceptor absorption spectra (see following sections). The spectral overlap ensures the resonance condition in EET, as illustrated by Fig. 1, which shows fluorescence transitions from D to multiple vibronic levels of the ground state, coupled to isoenergetic transitions of A. 
a)

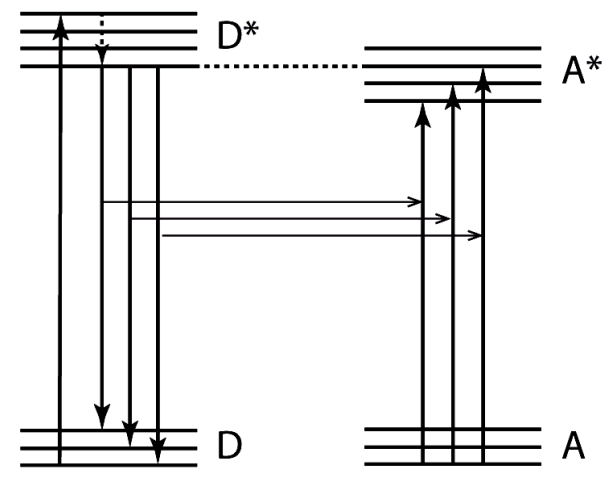

b)

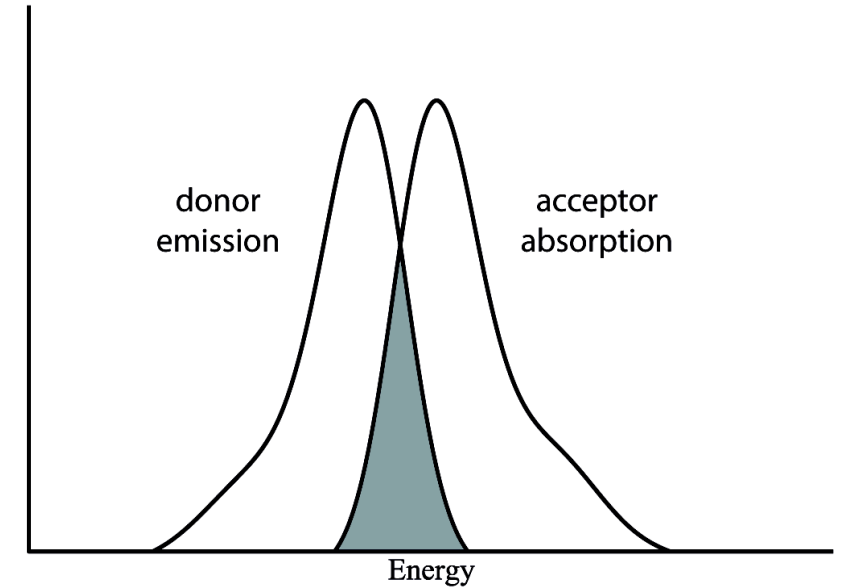

Figure 1: a) Schematic energy-level diagram of an energy transfer process. b) Spectral overlap between donor emission and acceptor absorption.

An alternative definition of the EET rate (1) is:

$$
k_{\mathrm{EET}}=k_{D}\left(\frac{R_{0}}{R}\right)^{6}=\frac{1}{\tau_{D}}\left(\frac{R_{0}}{R}\right)^{6}
$$

where $k_{D}=1 / \tau_{D}$ describes the decay rate of the excited $\mathrm{D}$ in the absence of $\mathrm{A}$ in terms of its lifetime $\tau_{D}$, and $R_{0}$ is the so-called critical quenching radius or "Förster radius", namely (in $\mathrm{nm}$ )

$$
R_{0}^{6}=\frac{9(\ln 10)}{128 \pi^{5} N_{A}} \frac{\left(\kappa^{2} \phi_{D}\right)}{n^{4}} I
$$

where $N_{A}$ is the Avogadro constant (in $\mathrm{mol}^{-1}$ ), $\phi_{D}$ is the fluorescence quantum yield, and $I$ the spectral overlap (in $\mathrm{M}^{-1} \mathrm{~cm}^{-1} \mathrm{~nm}^{4}$ ). Starting from this definition, the transfer efficiency can be expressed as

$$
\phi_{\mathrm{EET}}=\frac{k_{\mathrm{EET}}}{k_{D}+k_{\mathrm{EET}}}=\frac{1}{1+\left(R / R_{0}\right)^{6}}
$$

From Eqs. (5) and (7) it is evident that the Förster radius represents the distance where $k_{D}$ and $k_{\text {EET }}$ are equal and there is a $50 \%$ transfer efficiency.

The power of Förster theory, as given by Eq. (5) and (6), relies on the ability to relate D/A distances with transfer efficiencies directly from the spectroscopic parameters of the non-interacting D/A. Typically, transfer efficiencies are obtained from steady-state or timeresolved fluorescence measurements, so the FRET acronym is often referred to fluorescence(detected) resonance energy transfer, although this term could be confusing, as the transfer process itself is not mediated by fluorescence. ${ }^{12}$ 
In relating the efficiency with a $\mathrm{D} / \mathrm{A}$ distance, the main source of uncertainty in typical FRET experiments comes from the value adopted for the orientation factor $\kappa$. If both D and A molecules can freely rotate and are allowed to assume any mutual orientation during the lifetime of the donor, the isotropically averaged $\langle\kappa\rangle=2 / 3$ value can be used. This is often referred to as the "dynamic averaging regime", and is often fulfilled for small organic fluorophores attached with flexible linkers to a biomolecule. In some cases, however, the dynamic fluctuations of $\mathrm{D}$ and $\mathrm{A}$ cannot explore all possible orientations. In this case, fluorescence depolarization data can remove some uncertainty regarding the mutual D/A orientation. On the other hand, for large fluorophores like fluorescent proteins, rotational dynamics is expected to be slower than the D lifetime, giving rise to a heterogeneous ensemble characterized by different EET rates. In more complex cases, the timescales of rotational dynamics and excited-state decay are similar, which requires a more elaborated treatment of the data. Similar considerations apply to the D/A separation, for which fast fluctuations over a given distance mean that an average EET rate can be linked to the average $\langle R\rangle$ separation (dynamic averaging regime), whereas a static regime can occur if D and A are slowly diffusing, or EET occurs between slowly interconverting conformations.

Molecular dynamics (MD) simulations have proven to be very useful in generating possible conformations of the system that help interpreting observed efficiencies in terms of well-defined D/A separations and orientations. ${ }^{13-38}$ As it will be discussed in the following sections, MD simulations also allow investigating the interplay between EET and structural dynamics by properly post-processing trajectories of electronic coupling values, thus allowing to generate the expected FRET observables in intermediate situations between the limiting dynamic and static averaging regimes.

The point-dipole approximation (PDA) in Eq. (3) gives rise to the well-known $R^{-6}$ distance-dependence of the FRET rate. This approximation, however, breaks down at close D/A separations, when short-range effects and higher-multipole contributions become important. Moreover, it can only be applied to electronic transitions for which the transition dipoles are not null. Finally, the $1 / n^{2}$ dielectric screening factor assumes a homogeneous response of the surrounding environment, and neglects any possible dependence of the screening on mutual distance and orientation between $\mathrm{D}$ and $\mathrm{A}$. 
In the next section, we will discuss computational methods that overcome these fundamental limitations on the estimation of electronic couplings. Even with those accurate estimates of the coupling values, however, the Förster definition of the rate constant has other intrinsic limitations. The most fundamental one is the assumption that D and A are weakly coupled, on which the entire golden rule formulation of the rate, Eq. (1), is based. This assumption holds in an incoherent hopping regime, that is, when the coupling is much

smaller than the reorganization energy of the nuclei. ${ }^{39}$ In addition, it assumes a complete equilibration of the donor excitation prior to the transfer event. Finally, the spectral overlap factor also involves the assumption that the whole spectral broadening is homogeneous and that $\mathrm{D}$ and $\mathrm{A}$ are coupled to independent nuclear vibrations. In the following sections, we will also discuss issues related to the calculation of the spectral overlap factor, as well as quantum dynamics theories that can be applied to describe the EET rate in the intermediate and strong coupling regimes. These two situations are common in biomacromolecules organized as closely-packed multichromophoric systems, like photosynthetic antennae or nucleic acids.

\section{CALCULATION OF ELECTRONIC COUPLINGS}

In general, the electronic coupling should be written as a sum of a long-range (Coulomb) and a short-range contribution, the latter being dependent on the degree of orbital overlap between $\mathrm{D}$ and $\mathrm{A}:{ }^{40}$

$$
V=V_{\text {Coul }}+V_{\text {short }}
$$

Short-range terms are usually negligible, but they become dominant when the transfer involves spin-forbidden transitions; in these cases, the alternative electron exchange mechanism formulated by Dexter some years after Förster is used. ${ }^{41}$ An example where short-range terms become dominant is in triplet-triplet energy transfer (TEET), which is an overall spinallowed process, as the spin is conserved between the initial $\left({ }^{3} D^{* 1} A\right)$ and final $\left({ }^{1} D^{3} A^{*}\right)$ state, but the spin-forbidden nature of the involved transitions nullifies $V_{\text {Coul }}$.

For the most common case of spin-allowed transitions, electronic couplings are dominated by the Coulomb contribution, which reduces to the dipole-dipole term at large distances. 
The Coulomb interaction is strongly screened by a polarizable environment surrounding the donor and acceptor, an effect described through the $1 / n^{2}$ screening factor in Förster theory, as indicated in Eq. (2). When molecules are at distances comparable to their molecular dimensions, however, higher multipole contributions lead to large deviations from the dipoledipole interaction. ${ }^{42}$

In a symmetric homodimer, with identical D and A molecules, the electronic coupling can be estimated as one-half the energy splitting of the two electronic states due to their interaction. This "supermolecule" approach assumes that only one state of the donor and one of the acceptor mix to generate the two electronic states of the dimer, and it cannot be easily applied to asymmetric systems. ${ }^{8,43}$ In the following, we discuss more efficient and flexible strategies, which allow computing the Coulomb and the environment-mediated screening contributions from the transition properties of the non-interacting D and A fragments, as well as diabatization methods that allow estimating both Coulomb and short-range contributions in general asymmetric systems from dimer calculations.

\section{Coulomb contribution}

The (unscreened) coupling between two "bright" singlet excitations is dominated by the Coulomb interaction between the transition densities of the donor and the acceptor: ${ }^{40,44}$

$$
V_{\text {Coul }}=\int \mathrm{d} \mathbf{r} \int \mathrm{d} \mathbf{r}^{\prime} \rho_{D}^{T *}\left(\mathbf{r}^{\prime}\right) \frac{1}{\left|\mathbf{r}-\mathbf{r}^{\prime}\right|} \rho_{A}^{T}(\mathbf{r})
$$

where the transition density $\rho_{(A / D)}^{T}$ is the diagonal part of the one-particle density matrix constructed from the ground and excited-state wave functions:

$$
\begin{gathered}
\gamma_{0 i}^{T}\left(\mathbf{r}, \mathbf{r}^{\prime}\right)=N \int \ldots \int \psi_{i}^{*}\left(\mathbf{r}, \mathbf{r}_{2} \ldots \mathbf{r}_{N}\right) \psi_{0}\left(\mathbf{r}^{\prime}, \mathbf{r}_{2} \ldots \mathbf{r}_{N}\right) \mathrm{d} \mathbf{r}_{2} \mathrm{~d} \mathbf{r}_{3} \ldots \mathrm{d} \mathbf{r}_{N} \\
\rho_{0 i}^{T}(\mathbf{r})=\gamma_{0 i}^{T}(\mathbf{r}, \mathbf{r})
\end{gathered}
$$

By definition, the total integrated transition density is zero. Thus, the first nonzero term in a multipole expansion of the Coulomb coupling is that given by the interaction of the transition dipole moments. In transfers that involve dipole-forbidden transitions, the coupling can be dominated, for example, by dipole-quadrupole or higher-order terms. ${ }^{45}$ Moreover, it is well known that the PDA breaks down at "close" separations, when the details 
of the molecular shape become important. A rule of thumb to define "close" is the spatial dimension of the molecules, e.g., about 10-20 $\AA$ for small organic chromophores. Indeed, for typical fluorophores used in FRET experiments, it has been found that the PDA performs well at distances up to $20 \AA$, provided that the molecules sample an isotropic set of relative orientations. This performance, however, benefits from cancellation of errors due to isotropic motions. If such motions are restricted, significant errors can be found at distances as far as $50 \AA .{ }^{46}$ Similar deviations at considerable distances have also been found for interactions between pigments in a variety of photosynthetic complexes. ${ }^{42}$

In order to overcome the limitations of the PDA, a more robust strategy relies on computing the Coulomb coupling from the transition densities pre-calculated by some quantum mechanical (QM) method for the non-interacting D and A molecules. ${ }^{47}$ Early calculations of the Coulomb coupling were based on semi-empirical Hamiltonians. ${ }^{48-51}$ The first ab initio calculations, based on the configuration interaction with single excitations (CIS) method or time-dependent density functional theory (TD-DFT), ${ }^{52,53}$ adopted the so-called Transition Density Cube (TDC) method, where the integral in Eq. (9) is evaluated numerically over two separate three-dimensional grids representing the transition densities of A and D. A more recent and more computationally efficient strategy computes the integral in Eq. (9) via an atomic orbital expansion of the transition densities. ${ }^{47}$ This strategy, for example, has been used in combination with different QM methods such as CIS, TD-DFT, complete-activespace self-consistent-field (CASSCF), second-order approximate coupled cluster (CC2) and equation of motion coupled cluster (EOM-CC). ${ }^{54-56}$

An alternative way of computing Eq. (9) that has found widespread use relies on the transition charge approximation ( $\mathrm{TrCh}$ ), where the transition densities are represented by a set of point charges located on the atoms of D and A, respectively. Within this framework, the Coulomb coupling is expressed as the interaction between the D and A transition charges:

$$
V_{\mathrm{Coul}}^{\mathrm{TrCh}}=\sum_{K, L} \frac{q_{D}(K) q_{A}(L)}{\left|\mathbf{R}_{K}-\mathbf{R}_{L}\right|}
$$

where the indices $K$ and $L$ run on the $\mathrm{D}$ and A atomic positions $\mathbf{R}_{K}$ and $\mathbf{R}_{L}$, respectively. Various definitions of the atomic transition charges have been proposed so far, such as that based on a Mulliken population analysis of the transition densities. ${ }^{49,57-59}$ A more accurate 
definition of transition charges is the one based on the fitting to the electrostatic potential generated by the transition density, as it is typically done to parametrize point charge models in biomolecular force fields. This strategy, proposed by Renger and co-workers, is known as TrESP (transition charge from electrostatic potential), ${ }^{60}$ and represents a widely used method, in particular when coupled with MD simulations. ${ }^{61,62}$ Commonly, TrESP charges are computed once, at an optimized geometry, and used to compute couplings at different geometries. This scheme is computationally very efficient but it neglects any possible dependence of the transition density on the geometry. The TrESP approximation inevitably leads to a loss of accuracy if compared to the rigorous calculation of Eq. (9), especially at short distances. ${ }^{63,64}$ Nevertheless, it can be sistematically improved by adding higher order atomic multipoles to better fit the electrostatic potential. ${ }^{65,66}$

\section{Environment effects}

The environment is expected to strongly reduce the Coulomb interaction between D/A transition densities. This important screening effect is described through the $1 / n^{2}$ factor in the Förster expression (2) of the coupling, which leads to a four-fold attenuation of the EET rate in typical biological environments, where $n^{2}$ can be approximated to $2,{ }^{67}$ e.g. to the optical component of the dielectric constant of the medium $\left(\varepsilon_{\mathrm{opt}}\right)$. This reduction of the dipoledipole coupling was obtained by Forster by assuming a continuum dielectric embedding the two transition dipoles.

A more refined continuum model can be obtained by assuming that the donor and acceptor are placed in spherical cavities inside the dielectric: within this framework, a screening factor ranging from $3 /\left(2 \varepsilon_{\mathrm{opt}}+1\right)$ for dipolar transitions to $2 /\left(\varepsilon_{\mathrm{opt}}+1\right)$ for high-order multipoles is obtained. ${ }^{44}$ Even more realistic descriptions can be obtained by coupling the QM description of the chromophores to a modern continuum solvation model, like the Polarizable Continuum Model (PCM), in which the molecules are embedded in molecular-shaped cavities. ${ }^{68}$ Within this framework, the polarization of the dielectric is represented in terms of induced charges appearing on the molecular cavity surface. When applied to the calculation of the EET coupling, the PCM model leads to a further solvent-mediated contribution to be 
added to the (unscreened) Coulomb coupling in Eq. (9): ${ }^{47}$

$$
V_{\mathrm{PCM}}=\sum_{k} \int \mathrm{d} \mathbf{r}^{\prime} \rho_{D}^{T *}\left(\mathbf{r}^{\prime}\right) \frac{1}{\left|\mathbf{r}^{\prime}-\mathbf{s}_{k}\right|} q_{k}^{P C M}\left(\rho_{A}^{T}\right)
$$

This term represents the interaction between the transition localized at the donor site (and here represented by the transition density $\rho_{D}^{T}$ ) and the PCM surface charges $q^{\mathrm{PCM}}$ induced by the transition localized at $\mathrm{A}$ (represented by the transition density $\rho_{A}^{T}$ ); the same result is obtained by exchanging D and A. To obtain Eq. (13) it has been assumed that only the electronic component of the solvent polarization (determined by $\varepsilon_{\text {opt }}$ ) can be activated in the process. The Poisson-TrESP method reported by Renger and co-workers uses a similar expression based on the TrESP approach, in which the Poisson equation is solved for the electrostatic potential of the transition charges embedded in molecular-shaped cavities inside the dielectric. ${ }^{69,70}$

Application of the PCM model to a set of 100 pigment pairs extracted from the PE545, PC645, LHCII and PSII photosynthetic complexes showed that screening effects can strongly depend on the distance and mutual orientation between the chromophores, leading to an exponential attenuation of screening effects at close separations below $20 \AA .{ }^{71,72}$ This behaviour occurs when the pigments start to form a common cavity inside the dielectric, whose exposed surface is significantly reduced compared to the previously-separated individual cavities. However, note than in D/A pairs in which this cavity change is small, screening effects are similar at contact separations. ${ }^{72}$ Application of the Poisson-TrESP approach to a variety of pigment-protein complexes have also found a strong dependence of screening effects on relative $\mathrm{D} / \mathrm{A}$ orientations, but a weaker dependence on distance. ${ }^{70,73,74}$

An analogous expression for the solvent-dependent component to the coupling can be obtained within an atomistic (but still polarizable) model of the environment, such as when we introduce a polarizable Molecular Mechanics (MMPol) embedding. In fact, if we describe the environment as a set of fixed atomic charges (or a fixed multipolar expansion) and atomic polarizabilities, Eq. (13) becomes: ${ }^{75}$

$$
V_{\mathrm{MMPol}}=-\sum_{l} \int \mathrm{d} \mathbf{r}^{\prime} \rho_{D}^{T *}\left(\mathbf{r}^{\prime}\right) \frac{\left(\mathbf{r}^{\prime}-\mathbf{r}_{l}\right)}{\left|\mathbf{r}^{\prime}-\mathbf{r}_{l}\right|^{3}} \cdot \boldsymbol{\mu}_{l}^{\mathrm{MMPol}}\left(\rho_{A}^{T}\right)
$$

which represents the interaction between the transition density of D and the dipoles $\boldsymbol{\mu}^{\mathrm{MMPol}}$ induced on each polarizable site of the environment by the transition density of A. By 
introducing such an atomistic detail, the method can properly describe all the heterogeneities of the environment, ${ }^{76}$ which are instead not accounted for in a continuum model like PCM. Recently, this atomistic model has also been combined with a TrESP description of the D/A transitions. ${ }^{77}$

Both PCM and MMPol contributions indicated in Eqs. (13) and (14) describe a Coulomb coupling mediated by the surrounding environment, which typically reduces the overall interaction. In this context, one can define a screening factor from the ratio of the screened and unscreened Coulomb couplings, and an effective dielectric constant to compare $s$ with the Förster $1 / \varepsilon_{\text {opt }}$ screening factor:

$$
s=\frac{1}{\varepsilon_{\mathrm{eff}}}=\frac{V_{\mathrm{Coul}}+V_{\mathrm{env}}}{V_{\mathrm{Coul}}}
$$

Applications of the MMPol method to the PE545, ${ }^{76} \mathrm{FMO},{ }^{78} \mathrm{CP} 29,{ }^{79}$ and $\mathrm{LH} 2{ }^{80}$ photosynthetic complexes, to nucleic acids, including RNA $\beta$-hairpins, B-DNA, Z-DNA and DNA G-quadruplexes, ${ }^{81,82}$ or to protein-ligand complexes, ${ }^{83}$ indicate that the screening factors averaged over many pigment pairs are similar to those obtained adopting a continuum dielectric model and an $\varepsilon_{\text {opt }}=2$ value, as commonly assumed in biosystems. However, important deviations are observed for some pigment pairs, and effective dielectric constants ranging between 1 and 3 have been reported, depending on the particular orientation among the pigments and the surrounding environment atoms. This effect can be analyzed by inspecting the contribution of each MMPol atom to the summation in Eq. (14), as shown in the right panels of Figure 2 for some representative systems. In particular, in Figure 2c,d we show the two BChl pairs in the LH2 photosynthetic complex, which present completely different screening factors, depending on the position of the His residues with respect to the BChl planes. ${ }^{80,84}$ In addition, structural dynamics of a particular D/A pair can induce large fluctuations of the screening factor (See Figure 2). Whereas these fluctuations have been found to be small for photosynthetic complexes, large fluctuations on $s$ values in the range $0.4-0.8$ have been observed for the drug flurbiprofen interacting with Trp214 of human serum albumin (Figure 2a) due to a remarkable flexibility in the binding pocket. ${ }^{83}$

Overall, these results indicate that particular orientations, distances, and local environments can lead to important deviations from the $1 / \varepsilon_{\text {opt }}$ screening factor assumed in Förster 
theory and thus also in its distance and mutual D/A orientation dependence. In biomacromolecules, where the environment is significantly heterogeneous, correct screening effects can only be obtained with a polarizable and atomistic model for the environment.

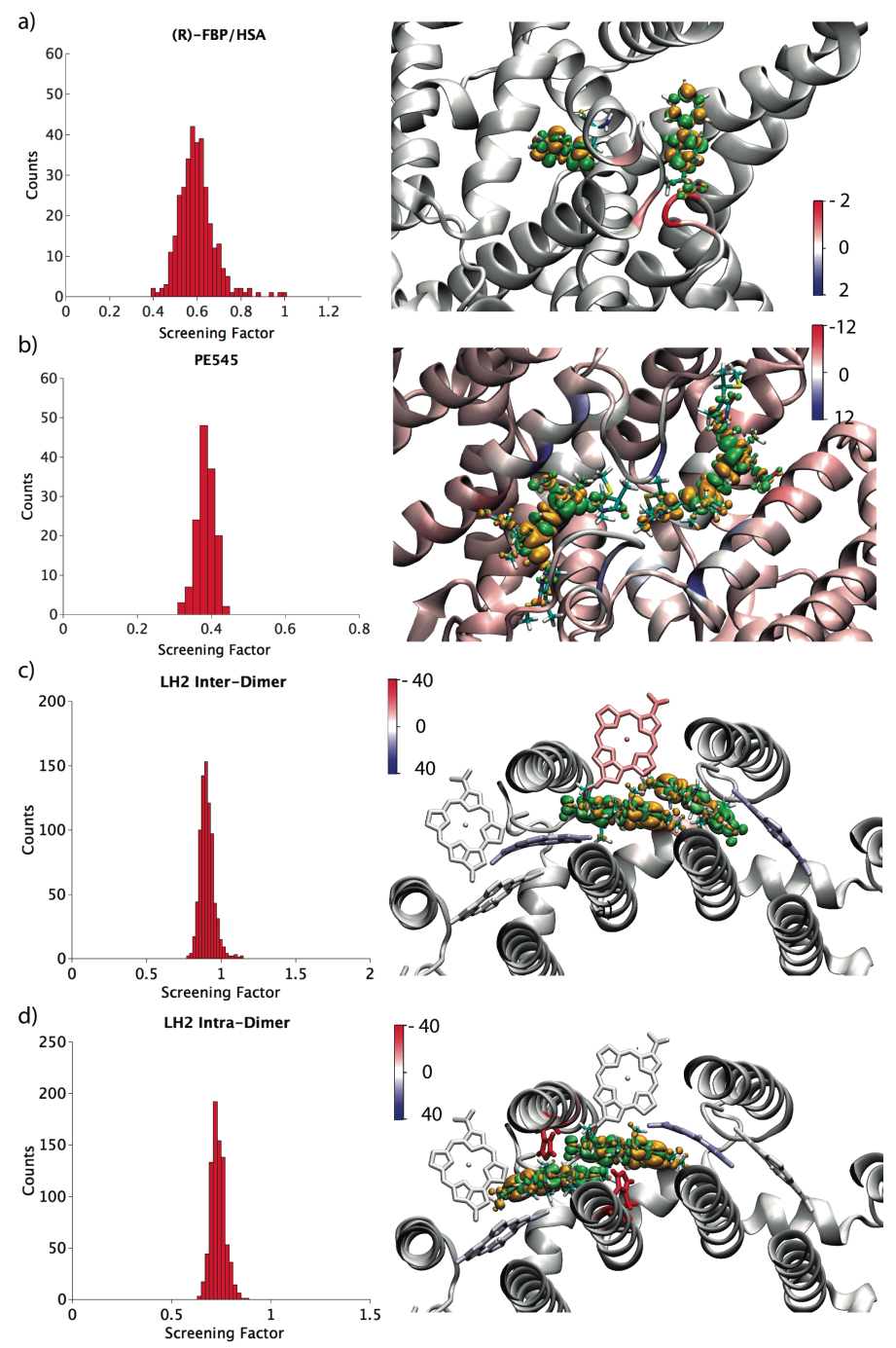

Figure 2: Distribution of dielectric screening factors estimated using the MMPol model along an MD simulation for different biosystems (left panels) and representation of chromophore transition densities and corresponding residue contributions (in $\mathrm{cm}^{-1}$ ) to the environment-mediated coupling term (right panels). a) (R)-flurbiprofen and Trp214 in human serum albumin (HSA), ${ }^{83}$ b) PEB50/61C and PEB50/61D bilin pigments in the PE545 complex, ${ }^{76}, \mathrm{c}$ ) inter-dimer and d) intra-dimer $\alpha$-BChl - $\beta$-BChl couplings in the B850 ring of the LH2 complex. ${ }^{80,84}$

Beyond classical multiscale methods, environment screening effects can also be estimated 
using the extension of subsystem TD-DFT to coupled excitations, in which the couplings between excitations localized in different subsystems can be selectively activated. ${ }^{85,86}$ In a comparison between this method and MMPol, a good agreement in terms of screening factors was found between the two methods, once a sufficiently large number of solvent excited states were coupled to the transitions of the dimeric systems studied. Similarly, if electronic couplings are derived from supermolecule calculations using a classical solvation model, screening effects can only be captured if the model includes the environment polarization response to the transition density.

\section{Short-range contribution}

As commented before, short-range contributions to the singlet EET are generally negligible as the Coulomb interaction is dominant. On the contrary, in triplet EET, where $V_{\text {Coul }}$ vanishes, only the short-range terms survive. Different QM methods have been proposed to evaluate these terms. A first-order perturbative expression was developed by Hsu et al. within a TD-DFT framework. In this approximation, the short-range terms include both an exchange-correlation and an overlap integral: ${ }^{44}$

$$
\begin{gathered}
V_{\text {short }}=V_{x c}+V_{\text {ovlp }} \\
V_{x c}=\int \mathrm{d} \mathbf{r} \int \mathrm{d} \mathbf{r}^{\prime} \rho_{D}^{T *}\left(\mathbf{r}^{\prime}\right) g_{x c}\left(\mathbf{r}^{\prime}, \mathbf{r} ; \omega_{0}\right) \rho_{A}^{T}(\mathbf{r}) \\
V_{\text {ovlp }}=-\omega_{0} \int \mathrm{d} \mathbf{r} \rho_{D}^{T *}(\mathbf{r}) \rho_{A}^{T}(\mathbf{r})
\end{gathered}
$$

where $g_{x c}$ is the exchange-correlation kernel of the selected DFT functional and $\omega_{0}$ is the common excitation energy of $\mathrm{D}$ and $\mathrm{A}$ molecules. The $x c$ term is replaced with the Dexter exchange integral if a HF/CIS description is adopted. ${ }^{40}$ Both exchange and overlap terms decay exponentially with the distance.

A more accurate strategy to evaluate short-range contributions relies on a diabatization strategy. Starting from a supermolecule calculation of the excited states in the D/A system, the resulting electronic Hamiltonian is finally transformed into the diabatic basis to extract the coupling. ${ }^{43}$ Within this context, the main issue is the definition of the diabatic states

for the energy transfer process. ${ }^{87}$ Different approaches have been proposed so far which are 
based on wave function or molecular properties, ${ }^{88-90}$ or constraints imposed on the electron density through constrained density functional theory (C-DFT). ${ }^{91}$ Some of these diabatization strategies rely on additional operators to define diabatic states. ${ }^{9,43}$ For example, the Fragment Excitation Difference (FED) method ${ }^{92}$ which is based on the Fragment Charge Difference method developed for electron transfer, ${ }^{93}$ defines the diabatic states as the linear combination of the D/A eigenstates that maximizes the degree of "exciton localization". The additional excitation difference operator $(\Delta x)$ is given in terms of "excitation densities", defined as the sum of attachment (electron) and detachment (hole) densities:

$$
\begin{gathered}
\Delta x_{m n}=\int_{r \in D} \rho_{m n}^{e x}(r) \mathrm{d} \mathbf{r}-\int_{r \in A} \rho_{m n}^{e x}(r) \mathrm{d} \mathbf{r} \\
\rho_{m n}^{e x}(r)=\rho_{m n}^{\operatorname{Det}}(r)+\rho_{m n}^{\mathrm{Att}}(r)
\end{gathered}
$$

Assuming a two-state approximation, diagonalization of the FED matrix leads to maximally localized excitations and to the following coupling expression:

$$
V^{\mathrm{FED}}=\frac{\left(E_{n}-E_{m}\right)\left|\Delta x_{m n}\right|}{\sqrt{\left(\Delta x_{m m}-\Delta x_{n n}\right)^{2}+4\left(\Delta x_{m n}\right)^{2}}}
$$

where $E_{n}$ and $E_{m}$ are the energies of the starting adiabatic states. The $V^{\mathrm{FED}}$ coupling includes both Coulomb and short-range contributions, as it is based on eigenstate properties. The FED method has been extended to triplet EET in the Fragment Spin Difference (FSD) scheme, in which the excitation density is replaced by a spin density, defined as the difference between $\alpha$ and $\beta$ electron densities. ${ }^{94}$ A more general approach, denoted Fragment Transition Density (FTD), has been introduced by Voityuk, ${ }^{95}$ which overcomes some limitations of the FED method, bypassing the definition of the excitation density of Eq. (20), and thus allowing one to use any QM level of theory that provides transition densities.

\section{The choice of the QM method}

The calculation of electronic couplings is quite robust regarding the choice of QM method and basis set, ${ }^{54,57,58,96,97}$ contrary to what generally observed for transition energies. In spite of this robustness, some issues have to be carefully checked in the selection of the QM description. 
In Figure 3 we show a comparison between mean percent errors in singlet and triplet EET couplings computed using different QM methods and basis sets.

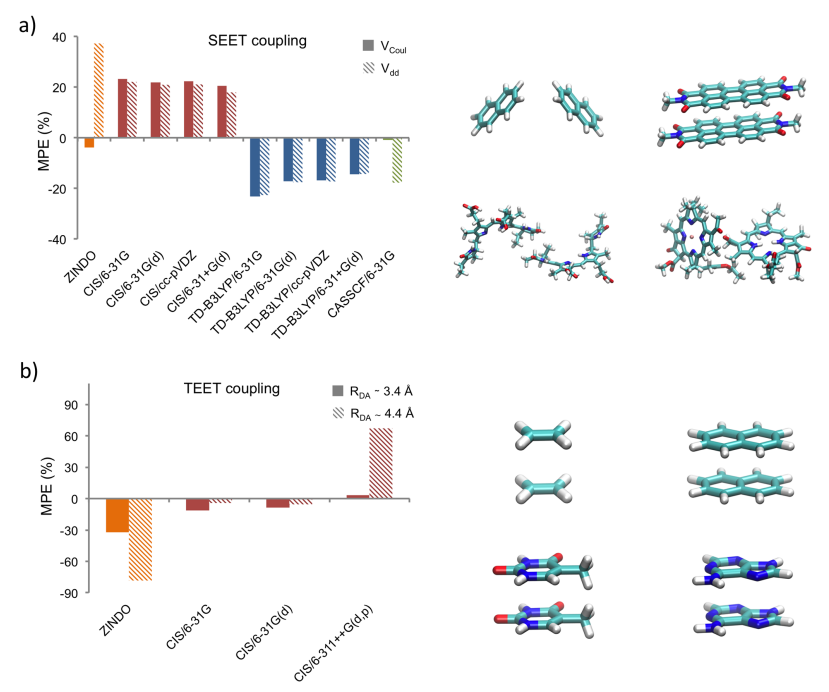

Figure 3: Mean percent error in absolute electronic coupling computed at different levels of theory for selected systems. a) Singlet EET Coulomb couplings for napthalene, perylene, PEB and BChl - BPheo pairs computed from QM transiton densities $\left(V_{\text {Coul }}\right)$ and dipoles $\left(V_{d d}\right)$ compared to SAC-CI/6-31G reference values. ${ }^{54}$ b) Triplet EET couplings for ethylene, napthalene, thymine and adenine pairs computed using the supermolecule approach compared to EOM-CCSD/6-31G reference values. ${ }^{98}$

Looking first to singlet EET (SEET), the data are shown for a napthalene, a perylene, a phycoerythrobilin (PEB) and a bacteriochlorophyll a (BChl) - bacteriopheophytin (BPheo) pair of chromophores. ${ }^{54}$ The PEB pair is found in the PE545 antenna of cryptophyte alga Rhodomonas CS24, whereas the BChl - BPheo pair corresponds to the reaction center of the purple photosynthetic bacterium Rhodobacter sphaeroides. The results correspond to Coulomb couplings computed either from the full transition densities $\left(V_{\text {Coul }}\right)$ or from transition dipoles $\left(V_{d d}\right)$. Both are compared to SAC-CI/6-31G $V_{\text {Coul }}$ and $V_{d d}$ reference values. From graph a), it is clear that the basis set has a rather small impact on the calculated couplings which be explained in terms of a weak dependence of the computed transition dipole strengths on the basis set. The choice of the QM method is instead much more important, as the length of the predicted dipoles is clearly method-dependent. This is illustrated by the errors estimated for $V_{d d}$ values, which show a systematic overestimation for the ZINDO 
and CIS methods, while TD-DFT and CASSCF calculations tend to underestimate them. If we compare the $V_{d d}$ and $V_{\text {Coul }}$ errors, however, we see that, for CIS and TD-DFT, they are very similar, suggesting that the difference between CIS and TD-DFT results compared to the reference SAC-CI values mostly arise from the transition dipole lengths. In these cases, an effective strategy to achieve more accurate couplings relies on a rescaling which uses the ratios between the experimental estimates of the D and A transition dipoles and the computed ones. ${ }^{52}$ Of course, such scaling is unable to correct inaccuracies in the orientation of the transition dipole moment or in the overall shape of the three-dimensional transition densities. The correspondence between $V_{d d}$ and $V_{\text {Coul }}$ errors is instead lost for the ZINDO values, and, to a lower extent, also for CASSCF results. With the exception of the semiempirical method, nevertheless, the errors arising from the choice of QM method on SEET couplings are relatively small, around $20 \%$.

The triplet EET couplings, on the other hand, display a quite different behaviour. In graph b) we show TEET couplings for stacked ethylene, napthalene, thymine and adenine pairs computed using the supermolecule approach compared to EOM-CCSD/6-31G reference values. In addition to the stacked complexes, with a $\mathrm{D} / \mathrm{A}$ separation $\mathrm{R} \sim 3.4 \AA$, we also reproduce results obtained at $\mathrm{R} \sim 4.4 \AA .{ }^{98}$ As expected, at $\mathrm{R} \sim 3.4 \AA$ the ZINDO method, based on the intermediate neglect of differential overlap (INDO) approximation, significantly underestimates by $30 \%$ the TEET coupling, whereas CIS and EOM-CCSD results are very similar, regardless of the basis set adopted. The intrinsic limitations of the ZINDO method, as well as the limitations of the basis sets adopted in CIS and EOM-CCSD calculations, are however drastically increased at $\mathrm{R} \sim 4.4 \AA$. In this case, the ZINDO calculations underestimate the EOM-CCSD/6-31G reference by $\sim 80 \%$. The results obtained at the CIS $/ 6-311++\mathrm{G}(\mathrm{d}, \mathrm{p})$ level, however, suggest that even the EOM-CCSD/6-31G reference values are underestimated by $\sim 70 \%$ due to the lack of diffuse functions, necessary to properly describe orbital overlap at this distance. Thus, whereas TEET couplings seem to show a much weaker dependence on QM method compared to singlet EET values, the choice of basis set can be rather important. 


\section{The role of fluctuations}

Because both Coulomb and short-range contributions are highly sensitive to the mutual distance and orientation between the $\mathrm{D}$ and $\mathrm{A}$ molecules, couplings can display large fluctuations, which are important to describe EET dynamics. The impact of these fluctuations can be illustrated by two limiting cases, the dynamic and the static averaging regimes. In the dynamic averaging regime, the coupling fluctuations are much faster than the donor lifetime. Thus, the rate can be expressed in terms of the averaged squared coupling value, $\left\langle V^{2}\right\rangle$. In the static averaging regime, in contrast, structural changes with timescales slower than the donor excited-state decay give rise to a distribution of rates corresponding to the different (static) structures. This problem has been widely discussed in the application of FRET to measure distances in biosystems, given that the main source of uncertainty is related to the value adopted for the orientation factor $\kappa \cdot{ }^{4}$ In this context, MD simulations have emerged as a powerful tool to examine the impact of electronic coupling fluctuations on FRET studies on a variety of biosystems and to aid in the characterization of the structural ensembles underlying static distributions of FRET rates. ${ }^{13-38}$ For example, simulations have shown some disagreement with the isotropically-averaged $\langle\kappa\rangle=2 / 3$ value often assumed in FRET studies, or the occurrence of significant correlations between distances and orientations, neglected in the isotropic factor by assuming that $\left\langle\kappa^{2} R^{-6}\right\rangle=\left\langle\kappa^{2}\right\rangle\left\langle R^{-6}\right\rangle \cdot{ }^{16} \mathrm{MD}$ simulations have also been used to analyze the role of coupling fluctuations on EET processes in biosystems where the D/A molecules are constrained to a given conformation, like photosynthetic systems, ${ }^{8}$ DNA $^{98,99}$ and protein-ligand complexes. ${ }^{83,100}$

Overall, simulation studies have shown that singlet and triplet EET couplings display qualitatively different fluctuations due to their different long- or short-range nature. In Figure 4 we show some examples of coupling fluctuations calculated using QM/MM methods along classical MD trajectories for different biosystems. The examples selected for singlet EET correspond to the flurbiprofen (FBP) - human serum albumin (HSA) complex ${ }^{83}$ and the interaction between two chlorophylls in the CP29 photosynthetic antenna, ${ }^{79}$ whereas triplet EET couplings are shown for two stacked adenines in $\mathrm{DNA}^{98}$ and between a chlorophyll and the violaxanthin carotenoid in CP29. ${ }^{101}$ TEET couplings display much larger variations 
than SEET values, as they are mediated by orbital overlaps, which strongly depend on the distance. Moreover, the distributions show tails toward large values. This means that computing TEET couplings for a static structure, for example a structure solved from X-ray crystallography, can introduce important errors in the prediction of TEET rates, ${ }^{101}$ and a better strategy relies on using MD-averaged coupling values, $\left\langle V^{2}\right\rangle$. Indeed, different MDbased studies have shown that structural fluctuations significantly enhance TEET dynamics, which can be explained on the basis of the tails observed in the coupling distributions. ${ }^{98,100,101}$

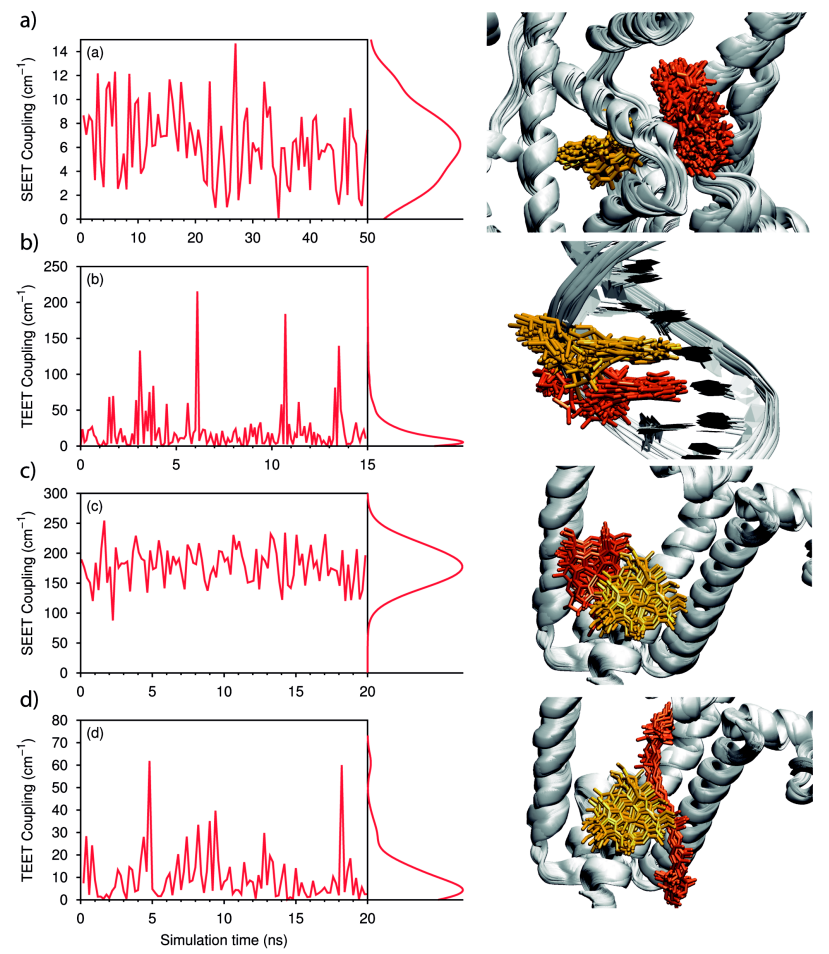

Figure 4: Electronic coupling fluctuations computed for different biosystems. Left panels show the time series of the couplings and the resulting distribution. Right panels show the geometrical fluctuations of the donor-acceptor pair that give rise to coupling fluctuations. a) SEET coupling between (R)-flurbiprofen and Trp214 in human serum albumin (HSA), ${ }^{83}$ b) TEET coupling between two adenines in a polyA-polyT DNA sequence, ${ }^{98}$ c) SEET coupling between chlorophylls a603 and a609 in the CP29 photosynthetic complex, ${ }^{79}$ d) TEET coupling between chlorophyll a603 and violaxanthin in the CP29 photosynthetic complex. ${ }^{101}$

Regarding SEET couplings, in systems where the D/A molecules are held in a relatively rigid conformation, for example the chlorophylls in the CP29 complex, the fluctuations dis- 
play a more gaussian distribution, as shown in Figure 4c, and a reasonable estimate of the rate can often be achieved by computing the coupling from a static structure. For example, a number of studies have been able to describe the light-harvesting properties of photosynthetic complexes based on static structures, although others adopt MD-averaged values. $8,102,103$ A recent study on the PE545 complex of cryptophyte algae, for example, showed that the explicit account of coupling fluctuations led to minor differences in EET dynamics compared to simulations based on the average value. ${ }^{104}$ If one of the chromophores displays considerable flexibility, however, even SEET couplings need to be averaged over structural fluctuations. This situation is exemplified in Figure 4a, which shows considerable fluctuations in the coupling between FBP and Trp214 of HSA. Indeed, the increased flexibility of (S)-FBP compared to (R)-FBP in the binding pocket was shown to explain the observed $\sim 40 \%$ faster EET dynamics for that enantiomer. ${ }^{83}$ On the other hand, the distribution of SEET couplings found for the FBP/HSA complex displays a less gaussian shape compared to the CP29 case, and the coupling values oscillate at slightly smaller values at longer simulation times, an indication of potential static coupling fluctuations.

In FRET systems where conformational transitions and fluorophore orientational dynamics can drastically change the mutual distance and orientation between the D/A chromophores, MD simulations are particularly useful in disentangling dynamic and static coupling fluctuations in the prediction of EET efficiencies. ${ }^{17,19,38}$ If the coupling fluctuations along the trajectory are fast compared to the donor excite-state decay (dynamic averaging regime), the efficiency can be calculated using the averaged rate constant:

$$
\phi_{\mathrm{EET}}=\left(1+\frac{K_{D}}{\left\langle k_{\mathrm{EET}}\right\rangle_{f}}\right)^{-1}
$$

In the opposite limit of slow coupling fluctuations (static averaging regime), there will be a distribution of energy transfer rates, and the ensemble efficiency can be computed by averaging the efficiency, rather than the rate, over static conformations:

$$
\phi_{\mathrm{EET}}=\left\langle\left(1+\frac{K_{D}}{k_{\mathrm{EET}}}\right)^{-1}\right\rangle_{s}
$$

When both dynamic and static fluctuations are relevant (intermediate averaging regime), the 
efficiency can thus be computed using a double average over static and dynamic disorder:

$$
\phi_{\mathrm{EET}}=\left\langle\left(1+\frac{K_{D}}{\left\langle k_{\mathrm{EET}}\right\rangle_{f}}\right)^{-1}\right\rangle_{s}
$$

Several groups have reported more elaborated approaches to describe EET in the intermediate averaging regime, in which Markov chain sampling techniques are used to model D (and eventually A) fluorescence decays in addition to FRET efficiencies from electronic coupling trajectories. ${ }^{22,24,25,28,29,38}$ In Scheme 1 we illustrate as an example the approach proposed by Grubmüller and co-workers. ${ }^{25}$ In this scheme, first a random donor excitation instance is chosen from the trajectory. Then, the Markov chain scheme is iterated in time steps $\Delta t=1$ ps until either photon emission or radiationless decay occurs in the $\mathrm{D}$ or $\mathrm{A}$ molecules. At each iteration, the rates for radiation or radiationless decay are assumed to be constant and derived from the lifetimes and quantum yields of $\mathrm{D}$ and $\mathrm{A}$, whereas the time-dependent EET rate $k_{\mathrm{EET}}(t)$ is computed from the electronic coupling in that point of the MD trajectory. Then, transitions are randomly selected based on the probabilities for thermal deexcitation $p_{\text {nonrad }, X}=k_{\text {nonrad }, X} \cdot \Delta t$, photon emission $p_{\text {rad }, X}=k_{\text {rad }, X} \cdot \Delta t$, EET $p_{\mathrm{EET}}(t)=k_{\mathrm{EET}}(t) \cdot \Delta t$, and no state change $1-p_{\text {nonrad }, X}-p_{\text {rad }, X}-p_{\mathrm{EET}}(t)$. In this scheme, the final EET efficiency can then be computed from the number of photons emitted by the donor and the acceptor.

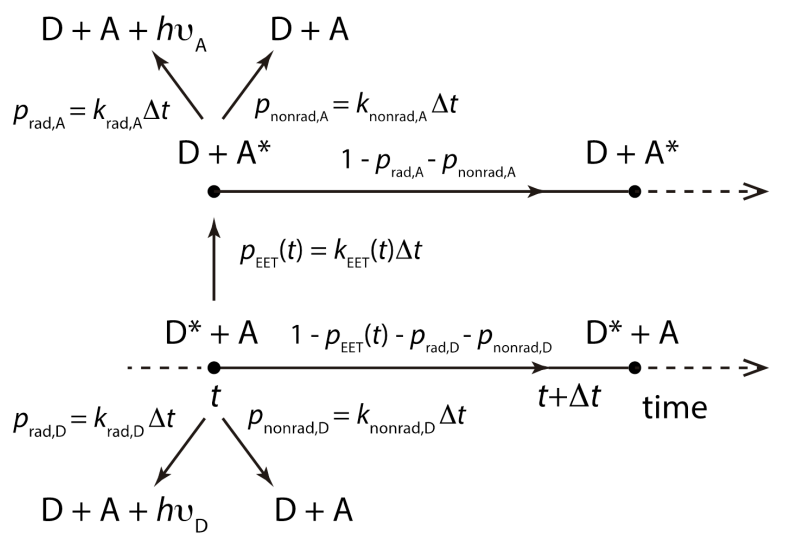

Scheme 1: Markov chain sampling technique used to model EET observables. 


\section{THE SPECTRAL OVERLAP}

\section{From the FCWD factor to the spectral overlap}

To derive the Fermi Golden rule for a molecular system, one has to consider two sets of vibronic states, $v$ and $v^{\prime}$, belonging respectively to the initial and final states $(i$ and $f)$. In this case, we have:

$$
k_{i f}=\frac{2 \pi}{\hbar}\left|V_{i f}\right|^{2} \sum_{v, v^{\prime}} p\left(E_{v}\right)\left|\left\langle v \mid v^{\prime}\right\rangle\right|^{2} \delta\left(E_{i v}-E_{f v^{\prime}}\right)=\frac{2 \pi}{\hbar}\left|V_{i f}\right|^{2} \mathrm{FCWD}
$$

where the summation runs over the vibrational states of $i$ and $f$, and contains the Boltzmann population $p\left(E_{v}\right)$ of the initial state $v$, and the Franck-Condon factors $\left|\left\langle v \mid v^{\prime}\right\rangle\right|^{2}$. The FCWD factor measures the probability that initial and final states have the same energy. Here, we have assumed the Condon approximation, which allows us to factorize the electronic coupling $V_{\text {if }}$ out of the summation.

In EET, the initial and final states are localized, respectively, on donor and acceptor. Assuming that each has its own independent vibrations, states $v$ are direct products of vibrational states of excited donor $v_{D}^{\prime}$ and ground-state acceptor $v_{A}$; likewise, $v^{\prime}$ are products of ground-state donor vibrational states $v_{D}$ and excited-state acceptor states $v_{A}^{\prime}$. The FCWD factor of Eq. (25) can thus be simplified by introducing the FCWD functions for donor emission and acceptor absorption:

$$
\begin{aligned}
f_{D}(E) & =\sum_{v_{D} v_{D}^{\prime}} p\left(E_{v_{D}^{\prime}}\right)\left|\left\langle v_{D}^{\prime} \mid v_{D}\right\rangle\right|^{2} \delta\left(E+\Delta E_{0 v_{D}, 1 v_{D}^{\prime}}\right) \\
f_{A}(E) & =\sum_{v_{A} v_{A}^{\prime}} p\left(E_{v_{A}}\right)\left|\left\langle v_{A} \mid v_{A}^{\prime}\right\rangle\right|^{2} \delta\left(E-\Delta E_{0 v_{A}, 1 v_{A}^{\prime}}\right)
\end{aligned}
$$

where $\Delta E_{0 v_{D}, 1 v_{D}^{\prime}}$ is the excitation energy of $\mathrm{D}$ from its ground state in the $v_{D}$ vibrational state to its excited state in the $v_{D}^{\prime}$ vibrational state, and the same definition is used for $\Delta E_{0 v_{A}, 1 v_{A}^{\prime}}$. The FCWD factor is finally expressed as the spectral overlap of the two separate FCWD functions of donor $\left(f_{D}\right)$ and acceptor $\left(f_{A}\right)$ :

$$
\mathrm{FCWD}=\int_{-\infty}^{\infty} \mathrm{d} E f_{D}(E) f_{A}(E)
$$


Notably, the functions $f_{A}(E)$ and $f_{D}(E)$ are related to the Franck-Condon expressions for acceptor absorption $A_{A}(\omega)$ and donor emission $F_{D}(\omega)$ spectra by the corresponding prefactors: $A_{A}(\omega) \propto\left|\mu_{A}^{t r}\right|^{2} \omega f_{A}(\hbar \omega)$, and $F_{D}(\omega) \propto\left|\mu_{D}^{t r}\right|^{2} \omega^{3} f_{D}(\hbar \omega)$. This allows computing spectral overlaps via Eq. (28) from experimental spectra, ${ }^{1}$ after dividing each spectrum by its frequency prefactor ( $\omega$ for the absorption and $\omega^{3}$ for the fluorescence), and normalizing the functions $f_{A}(E)$ and $f_{D}(E)$. In some cases, i.e. when the initial or final state is dark, or there are overlapping bands in the acceptor absorption, it becomes necessary to estimate the spectral overlap by calculating $f_{A}(E)$ and $f_{D}(E)$.

While it is possible to compute the FCWD functions from Franck-Condon calculations, the donor-acceptor energy difference must be estimated accurately, as spectral overlaps may be highly sensitive to this difference, and a small deviation in the excitation energies may result in a large error on the computed rate. ${ }^{105}$ As an example, we report in Figure 5 the spectral overlap as a function of the vertical excitation energy of the acceptor, while maintaining all other parameters fixed.

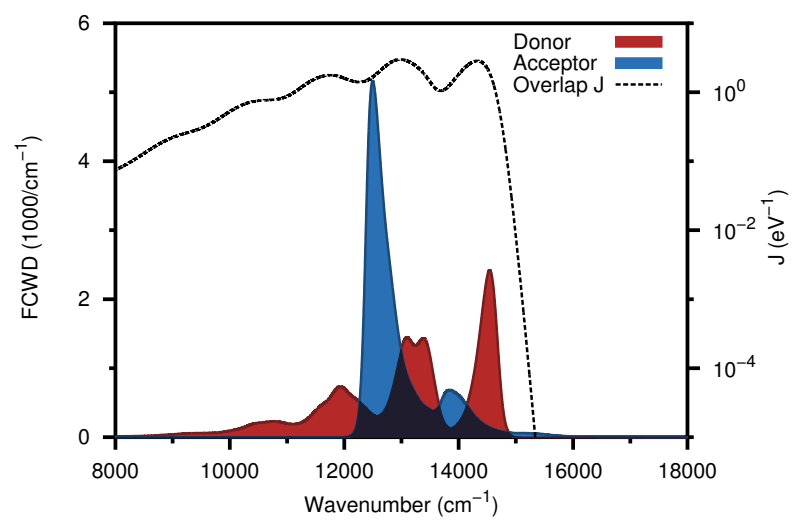

Figure 5: Spectral overlap between a carotenoid donor and a bacteriochlorophyll acceptor. The filled curves represent the densities of states $f_{A}$ and $f_{D}$, whilst the dashed line represents the value of the spectral overlap $J$ as a function of the vertical excitation energy of the acceptor, in logarithmic scale.

Sometimes, rather crude estimations of spectral overlaps are found in the literature, where the FCWD functions are replaced by gaussian functions whose position and width is estimated from experimental spectra. ${ }^{48,59}$ A more accurate fitting can be done by express- 
ing the FCWD function as a single-mode vibronic progression. When dealing with triplet states, the difficulty in obtaining triplet absorption spectra can be overcome by employing spectral parameters for singlet absorption, and adjusting the excitation energy to match the triplet energy. ${ }^{101,105}$ Particular caution should be used when determining spectral overlaps from experimental spectra, because their lineshape contains contributions from both homogeneous and inhomogeneous broadening. The latter is due to static disorder in the excitation energy and arises from an ensemble of molecules experiencing different environments. Each microstate of the ensemble is characterized by different excitation energies in both donor and acceptor, and therefore by the spectral overlap. An effective spectral overlap can be obtained by averaging over the inhomogeneous distributions of donor and acceptor energies, ${ }^{1,106}$ which is analogous to the static averaging discussed in the previous section. At room temperature, homogeneous broadening usually dominates the lineshape, and the distribution of rates due to the inhomogeneous energy distribution is rather small.

\section{Spectral density and time-domain formulation of the EET rate}

A very effective approach to obtain the FCWD is a time-domain expression based on the spectral density (SD) of the exciton-phonon coupling. ${ }^{107,108}$ The spectral density is defined as the Fourier transform of the quantum autocorrelation function (ACF) of the excitation energy fluctuations $\Delta E$, calculated on the ground-state potential energy surface (PES):

$$
\begin{aligned}
& C(\tau)=\langle\Delta E(\tau) \Delta E(0)\rangle \\
& \tilde{C}(\omega)=\int_{-\infty}^{\infty} e^{i \omega t} C(t) \mathrm{d} t=\tilde{C}^{\prime}(\omega)+\tilde{C}^{\prime \prime}(\omega)
\end{aligned}
$$

where $\tilde{C}^{\prime}(\omega)$ and $\tilde{C}^{\prime \prime}(\omega)$ refer to the even and odd parts of $\tilde{C}(\omega)$.

Since $C(\tau)$ is a quantum $\mathrm{ACF}$, it has both a real and an imaginary part. In the frequency domain, their Fourier transforms $\tilde{C}^{\prime}(\omega)$ and $\tilde{C}^{\prime \prime}(\omega)$ satisfy the detailed balance condition: ${ }^{107,108}$

$$
\tilde{C}^{\prime}(\omega)=\operatorname{coth}\left(\frac{\beta \hbar \omega}{2}\right) \tilde{C}^{\prime \prime}(\omega)
$$

As $\tilde{C}^{\prime}(\omega)$ and $\tilde{C}^{\prime \prime}(\omega)$ share the same amount of information, we can define the spectral density as just the odd part $\tilde{C}^{\prime \prime}(\omega),{ }^{108}$ from which all vibronic properties can be obtained, through 
the lineshape function $g(t)$ :

$$
\begin{aligned}
& g(t)=\int_{0}^{t} \mathrm{~d} \tau_{2} \int_{0}^{\tau_{2}} \mathrm{~d} \tau_{1}\left\langle\Delta E\left(\tau_{1}\right) \Delta E(0)\right\rangle \\
& =-\int_{0}^{\infty} \frac{\tilde{C}^{\prime \prime}(\omega)}{\pi \omega^{2}}\left[\operatorname{coth}\left(\frac{\beta \hbar \omega}{2}\right) \cdot(\cos (\omega t)-1)-i(\sin (\omega t)-\omega t)\right] \mathrm{d} \omega
\end{aligned}
$$

From $\tilde{C}^{\prime \prime}(\omega)$ we can also obtain the total coupling to vibrations. This can be quantified in terms of the reorganization energy $\lambda$, defined as:

$$
\lambda=\int_{0}^{\infty} \frac{\tilde{C}^{\prime \prime}(\omega)}{\pi \omega} \mathrm{d} \omega=-\lim _{t \rightarrow \infty} \frac{\mathrm{d}}{\mathrm{d} t} \Im\{g(t)\}
$$

Using the properties of the Fourier transform, the FCWD of Eq. (28) can be recast in the time domain and the EET rate rewritten as: ${ }^{109,110}$

$$
k_{E E T}=\frac{\left|V_{D A}\right|^{2}}{\hbar^{2}} \Re \int_{0}^{\infty} \exp \left\{-i \omega_{A D} t-2 i \lambda_{D} t-g_{A}(t)-g_{D}(t)\right\} \mathrm{d} t
$$

where $\lambda_{D}$ is the reorganization energy of the donor state, $g_{A}(t)$ and $g_{D}(t)$ are the lineshape functions of the donor and acceptor, and $\omega_{A D}$ is the difference between the vertical excitation energies of $A$ and $D$. Equation (33) can be obtained from a generalized master equation, by treating the electronic coupling $V_{D A}$ as a perturbation. ${ }^{74,110}$ The time-domain approach is useful to recover some of the approximations made in the derivation of the FCWD term. For example, if the modes of $A$ and $D$ are not independent, a new term $g_{A D}(t)$, that accounts for correlated energy fluctuations, appears in the exponent of Eq. (33). In this condition, the the FCWD term cannot be expressed as spectral overlap (see Eq. (28)), because now the integrand in the time domain cannot be factorized. ${ }^{111}$

A classical MD trajectory can be used to obtain the classical, real-valued ACF of the excitation energy $C_{\mathrm{cl}}(t)$, which corresponds to the even $\tilde{C}^{\prime}(\omega)$. The odd part can be recovered a posteriori, multiplying by a temperature-dependent prefactor, in the so-called Harmonic approximation: ${ }^{108}$

$$
\tilde{C}^{\prime \prime}(\omega)=\frac{\beta \omega}{\pi} \int_{-\infty}^{\infty} e^{i \omega t} C_{\mathrm{cl}}(t) \mathrm{d} t
$$

where $\beta=1 / k_{B} T$ depends on the temperature of the classical trajectory. The classical ACF is calculated from the excitation energies $\Delta E\left(t_{i}\right)$ along snapshots $t_{i}$ of the MD trajectory: ${ }^{108}$

$$
C_{\mathrm{cl}}\left(t_{j}\right)=\frac{1}{N-j} \sum_{i=1}^{N-j} \Delta E\left(t_{i}+t_{j}\right) \Delta E\left(t_{i}\right)
$$


Given the size and complexity of biological systems, spectral densities have been normally computed on the basis of classical MD simulations, where the nuclear trajectory is computed with a Molecular Mechanics (MM) force field, and the excitation energy evaluated on the resulting configurations using a quantum mechanical approach. Generally, the effect of the surrounding environment on the excitation energy fluctuations is taken into account by employing multiscale QM/MM models. This allows evaluating the coupling of the excitation to both the modes of the pigments and those of the surroundings.

In order to converge the calculation of $C_{\mathrm{cl}}(t)$, a reasonable sampling is needed. In order to sample high-frequency modes, the time step between the configurations used for the excitedstate calculations should be rather small ( $\lesssim 5 \mathrm{fs}),{ }^{108,112,113}$ while accurate sampling of lowfrequency modes (i.e. $\sim 30 \mathrm{~cm}^{-1}$, corresponding to a period of $\sim 1 \mathrm{ps}$ ) requires a time window of at least $10 \mathrm{ps}$, that is, long enough to sample several oscillations. Before computing the spectral density, $C_{\mathrm{cl}}(t)$ can be multiplied by a damping Gaussian or exponential function to ensure complete decay within the time window ${ }^{108}$. This is reflected in Gaussian or Lorentzian broadening of the SD peaks.

It has been questioned as to whether the MD-based approach is accurate enough to describe excitation energy fluctuations, ${ }^{114,115}$ the most concerning issue being related to the quality of the underlying MM-based MD. In fact, the MM Hamiltonian used to generate the trajectory differs from the QM Hamiltonian used to compute the excitation energies, ${ }^{115-117}$ leading to inaccurate excitation energy fluctuations $\langle\Delta E(\tau) \Delta E(0)\rangle$. For example, a shifted equilibrium position of the MM PES with respect to the ground-state QM PES likely leads to exaggerated fluctuations, and to overestimating the SD peaks, ${ }^{116}$ whereas an incorrect curvature of the MM PES leads to errors in the position of the SD peaks and to a redistribution of the SD intensities. ${ }^{114}$ Indeed, SDs calculated with the MD-based approach are strongly dependent on the parameters of the force field, rather than on the QM method used to compute the excitation energies. ${ }^{112,118}$

The best solution to the force-field issue would be using a QM/MM MD to calculate the ground-state trajectory, ${ }^{119-121}$ thus retaining both the accuracy of the QM method for the chromophore and the effect of the surrounding environment on the ground-state PES. This approach is not always feasible, given the dimensions of biological chromophores and 
the long time window requested by the sampling (vide supra). A compromise solution can be the development of ad-hoc force-fields for the chromophores, specifically targeted to the description of excitation properties, ${ }^{122,123}$ possibly employing PESs directly interpolated from quantum chemical data. 117,124

An alternative approach to SD calculations is the normal-mode analysis proposed by Lee et al. ${ }^{114}$ and applied to FMO, phycobiliprotein complexes, ${ }^{125}$ and the LH2 system of

purple bacteria. ${ }^{126}$ Such an approach is based on the analytical expression of $\tilde{C}^{\prime \prime}(\omega)$ when the ground and excited state PESs are described by the same harmonic potential, but with shifted equilibrium position:

$$
C^{\prime \prime}(\omega)=\pi \sum_{k} \omega_{k} \lambda_{k} \delta\left(\omega-\omega_{k}\right)
$$

where $\omega_{k}$ and $\lambda_{k}$ are the frequency and the reorganization energy of the $k$-th normal mode. These quantities can be obtained through QM/MM frequency calculations, in which the chromophore is placed in a frozen environment. ${ }^{114,115}$ A subsequent calculation of the excitedstate gradient at the Franck-Condon point (vertical gradient, or VG) is used to determine the reorganization energies $\lambda_{k}=f_{k}^{2} / 2 \omega_{k}^{2}$, where $f_{k}$ is the vertical gradient along the direction of normal mode $k$. Usually, only the chromophore fluctuations are included in this model. The effect of protein fluctuations on the SD can be recovered by computing only solvatochromic shifts along an MD trajectory. ${ }^{114}$ However, the normal-mode approach does not necessarily require a molecular dynamics, and can be used directly on a single structure, ${ }^{126}$ even though in this case the effect of protein fluctuations can be only added a posteriori.

\section{BEYOND FÖRSTER}

The Förster formulation of EET, as described in the previous sections, is based on the assumption of excitations being localized on one chromophore at a time, while energy transfer occurs by incoherent "hops" between chromophores. 7,39,110,127 However, when the electronic coupling between donor and acceptor is large, the perturbative Golden Rule expression (1) is not valid anymore ${ }^{74,127}$, as the electronic coupling tends to delocalize the excitation over both chromophores, giving rise to an exciton state. In multichromophoric systems as DNA 
or light-harvesting pigment-protein complexes, the excitation may be shared among several chromophores. In these cases different formulations have to be introduced as summerized in the next sections.

\section{The generalized Förster approach}

If we assume that the multichromophoric system can be divided in two weakly connected aggregates, each containing multiple chromophores and that, within each aggregate, the electronic coupling is strong enough to delocalize the excitation, the EET rate can be recovered from a generalization of the Forster equation between new "exciton" states corresponding to the $\mathbf{D}$ and $\mathbf{A}$ aggregates.

Each "exciton" state $K$ can be written as an expansion over the site-localized states $j$ :

$$
|K\rangle=\sum_{j=1}^{n} c_{j K}|j\rangle
$$

where $n$ is the total number of chromophores in each aggregate. In the basis of the states $j$ (site basis), the electronic Hamiltonian of the aggregate is written as:

$$
H_{e l}=\sum_{j=1}^{n} \mathcal{E}_{j}|j\rangle\left\langle j\left|+\sum_{i \neq j}^{N} V_{i j}\right| i\right\rangle\langle j|
$$

where $\mathcal{E}_{j}$ is the excitation energy of chromophore $j$, and $V_{i j}$ is the electronic coupling between states $i$ and $j$. The coefficients $c_{j K}$ are obtained by diagonalization of $H_{e l}$, and the corresponding eigenvalues $E_{K}$ represent the excitation energies of the exciton states.

In order to generalize the rate equation (33) to describe EET between exciton states of D and $\mathrm{A}$, we need to define the lineshape tensors $g_{K L M N}(t)$ and reorganization energies $\lambda_{K L M N}$ for the exciton states: ${ }^{74,128}$

$$
\begin{aligned}
& g_{K L M N}(t)=\sum_{j=1}^{n} c_{j K}^{*} c_{j L} c_{j M}^{*} c_{j N} g_{j}(t) \\
& \lambda_{K L M N}=\sum_{j=1}^{n} c_{j K}^{*} c_{j L} c_{j M}^{*} c_{j N} \lambda_{j}
\end{aligned}
$$

Equation (39) shows that the vibrations of site $j$ are now shared among several exciton states. The lineshape function of $K$ is the diagonal part $g_{K} \equiv g_{K K K K}$ of the lineshape tensor, and the same is valid for the reorganization energy $\lambda_{K}$. 
The EET rate between exciton states $K \in \mathbf{D}$ and $L^{\prime} \in \mathbf{A}$ is described by the Generalized Förster (GF) rate equation: ${ }^{1,106,129}$

$$
k_{K L^{\prime}}=\frac{1}{\hbar^{2}}\left|\sum_{i \in \mathbf{D}} \sum_{j^{\prime} \in \mathbf{A}} c_{i K}^{*} c_{j L^{\prime}} V_{i j^{\prime}}\right|^{2} \Re \int_{0}^{\infty} \exp \left\{-i \omega_{K L^{\prime}} t-2 i \lambda_{K} t-g_{L^{\prime}}(t)-g_{K}(t)\right\} \mathrm{d} t
$$

where the non-primed indices run on $\mathbf{D}$ states, and the primed indices run on the $\mathbf{A}$ states. The effective coupling is obtained by transforming the coupling $V_{i j}$ in the basis of the ex-

citon states of $\mathbf{D}$ and $\mathbf{A}$, whereas the effective spectral overlap is expressed through the excitation energy difference $\hbar \omega_{K L^{\prime}}=\left(E_{K}-E_{L^{\prime}}\right)$, and through the lineshape functions and reorganization energies of exciton states.

Closely related to GF is the multichromophoric FRET (MC-FRET), which recovers exciton coherence terms in the effective spectral overlap by defining generalized absorption and emission tensors. ${ }^{130,131}$ Both GF and MC-FRET have been successfully applied to the LH2 system of purple bacteria to describe EET between the B800 BChls and the strongly coupled B850 ring. ${ }^{106,130}$

\section{Exciton relaxation}

When EET occurs within strongly coupled aggregates the GF formulation is no longer valid. The strong coupling regime is established when the electronic coupling is much larger than the coupling to vibrational degrees of freedom, which is quantified through the reorganization energy $\lambda$ (vide supra). ${ }^{39,74,132}$

Within the strong coupling limit, the excited-state dynamics of the system consists in relaxation between exciton states. The relaxation rate between states $K$ and $L$ can be expressed in the Redfield theory formulation as: ${ }^{74,110}$

$$
k_{K L}=\sum_{j=1}^{n}\left|c_{j K}\right|^{2}\left|c_{j L}\right|^{2} C_{j}^{\prime \prime}\left(\omega_{K L}\right)\left(1+\operatorname{coth}\left(\frac{\beta \hbar \omega_{K L}}{2}\right)\right)
$$

where $C_{j}^{\prime \prime}\left(\omega_{K L}\right)$ is the spectral density of site $j$ evaluated at the energy gap between states $K$ and $L$.

The standard Redfield (sR) rate equation (42) describes a relaxation process where one quantum of electronic energy $\hbar \omega_{K L}$ is released to the vibrational degrees of freedom through 
the vibronic coupling represented by the spectral density, and cannot treat processes where multiple energy quanta are transferred to the vibrations. ${ }^{110}$ An alternative formulation of the exciton relaxation rate is given by the modified Redfield $(\mathrm{mR})$ theory, which takes into account the FCWD factor between exciton states. The $\mathrm{mR}$ rate is given by: ${ }^{74,110,128,133}$

$$
k_{K L}=2 \Re \int_{0}^{\infty} \mathrm{e}^{-i \omega_{K L} t-2 i \lambda_{K} t-g_{K}(t)-g_{L}(t)} V_{K L}(t) \mathrm{d} t
$$

where $V_{K L}(t)$ is expressed as

$$
\begin{aligned}
& V_{K L}(t)=e^{2 g_{K K L L}(t)+2 i \lambda_{K K L L} t} \times \\
& \times\left\{\ddot{g}_{L K K L}(t)-\left(\dot{g}_{K L K K}(t)-\dot{g}_{K L L L}(t)+2 i \lambda_{K L K K}\right)\left(\dot{g}_{K K L K}(t)-\dot{g}_{L L L K}(t)+2 i \lambda_{K K L K}\right)\right\}
\end{aligned}
$$

Here, $\dot{g}$ and $\ddot{g}$ denote first and second time derivatives, and $g_{K L M N}(t)$ and $\lambda_{K L M N}$ are defined in Eq. (39). Notably, the modified Redfield equation (44) resembles the time-domain expression of the Förster rate (33), though here the "coupling" term $V_{K L}(t)$ is time-dependent and cannot be factorized out of the integral.

In the limit of large energy gap between donor and acceptor, initial and final states become localized on either site, and the Förster equation (33) is exact. In this regime, contrary to the sR rate, $\mathrm{mR}$ exactly recovers the Förster rate ${ }^{109,128}$. However, when donor and acceptor have the same energy, the $\mathrm{mR}$ rate reduces to the standard Redfield, and never recovers the Förster rate in the limit of weak coupling. ${ }^{109,128}$ 

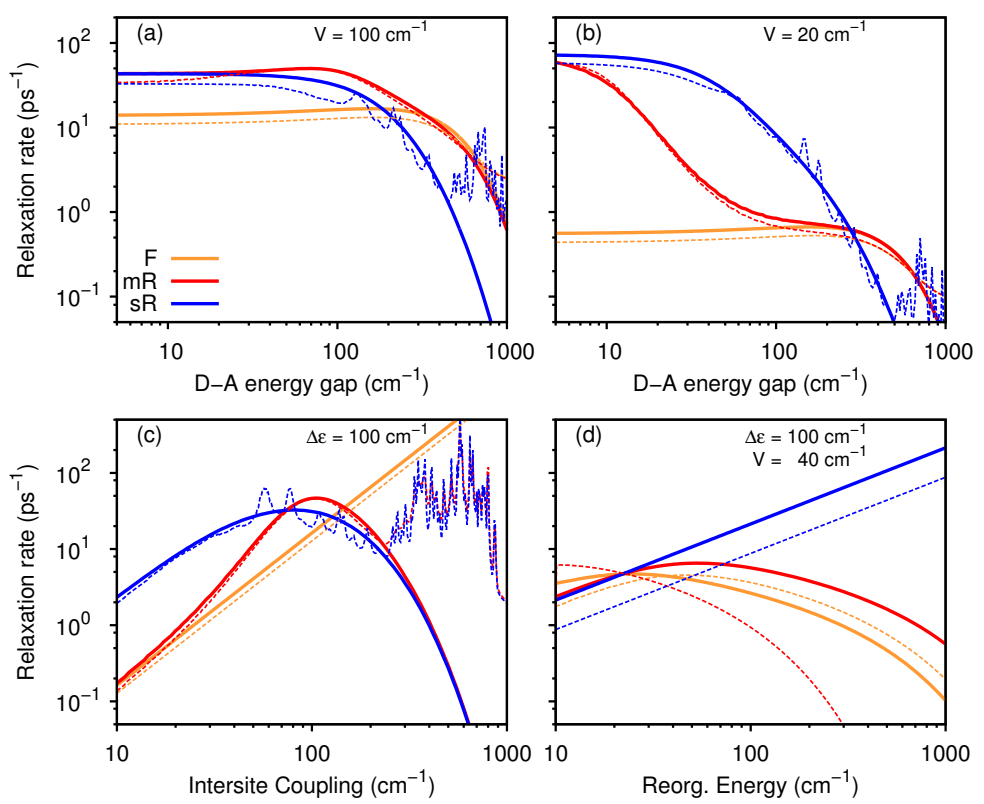

Figure 6: Downhill exciton relaxation rates in a D-A dimer, as predicted by the Förster equation (F, orange lines), modified Redfield theory (mR, red lines), and standard Redfield theory (sR, blue lines). Solid lines correspond to the model B777 SD, ${ }^{134}$ while dashed lines correspond to the calculated B800 SD including high-frequency vibrations. ${ }^{126}$ The two SDs have total reorganization energies of $\sim 100$ and $\sim 220 \mathrm{~cm}^{-1}$, respectively, and comparable reorganization energy for lower frequencies $\left(\lesssim 500 \mathrm{~cm}^{-1}\right)$. We used the same SD for donor and acceptor. In (a) and (b) we plot downhill rates as a function of the energy gap between $\mathrm{D}$ and $\mathrm{A}$, for electronic coupling values of (a) $V=100 \mathrm{~cm}^{-1}$ or (b) $V=20 \mathrm{~cm}^{-1}$. (c) Downhill rates as a function of the electronic coupling $V$, for energy gap $\Delta \mathcal{E}=100 \mathrm{~cm}^{-1}$. (d) Downhill rates as a function of the total reorganization energy of the SD. Here we use $\Delta \mathcal{E}=100 \mathrm{~cm}^{-1}$ and an intermediate coupling $V=40 \mathrm{~cm}^{-1}$. In all cases the temperature was fixed at $300 \mathrm{~K}$.

To better understand the above discussion, in Figure 6a,b we compare the standard and modified Redfield theories to the Förster rate equation (33) for varying energy gap between donor and acceptor. Following Yang and Fleming ${ }^{110}$, we selected two regimes of strong (100 $\left.\mathrm{cm}^{-1}\right)$ and weak $\left(20 \mathrm{~cm}^{-1}\right)$ coupling, but we modeled the coupling to vibrations with more realistic spectral densities: firstly, the so-called B777 SD fitted on high-resolution lowtemperature fluorescence spectra of BChl on the B777 pigment-protein complex (solid lines in the Figure). ${ }^{134}$ Dashed lines represent the SD computed with the normal-mode analysis 
on the B800 BChl of $\mathrm{LH}^{126}$, which contains several high-frequency peaks. In all cases, $\mathrm{mR}$ and sR become identical when the energy gap is much smaller than the electronic coupling. In the opposite limit, modified Redfield correctly recovers the Förster rate. As it regards the standard Redfield, the results obtained with the B777 SD closely resemble those discussed by Yang and Fleming on a different structureless SD and give very small rates. ${ }^{110}$ Conversely, the structured B800 SD gives non-vanishing sR rates also at larger energy gaps, due to the high-frequency SD peaks. The sR rates are exactly proportional to the SD intensity at the energy gap, and "follow" the structure of the SD. The mR rates instead show a smoother behavior with the energy gap, as $\mathrm{mR}$ theory allows relaxation processes that involve multiple vibrational quanta. Multi-phonon relaxation results in generally larger rates for $\mathrm{mR}$ theory, especially for larger gaps; however, in some regions the $\mathrm{mR}$ rates are smaller than the corresponding sR rates. In pigment-protein complexes, where the effective rates are averaged over the disorder and over many chromophores, modified and standard Redfield give similar results on rates and dynamics. ${ }^{74,126}$

Figure $6 \mathrm{c}$ shows the behaviour of $\mathrm{mR}$ and $\mathrm{sR}$ with increasing coupling, at a fixed energy gap. Here the Förster rate is proportional to the squared coupling, and unbound. Again, mR theory reduces to the correct Förster limit for weak coupling, contrary to sR, which overestimates the rates by one order of magnitude. However, we recall that, for near degeneracy between donor and acceptor, $\mathrm{mR}$ would overestimate the rates as $\mathrm{sR}$ does. ${ }^{128}$ The incorrect behaviour of both Redfield formulations for large exciton-vibrational coupling is exemplified in Figure 6d, where we compute the rates for scaled spectral densities in order to span a large interval of reorganization energies, for fixed energy gap and coupling. Here, the sR rate is always proportional to the reorganization energy, and grows without bounds. Even though the coupling and energy gap values make the excitons quite localized, $\mathrm{mR}$ theory fails to recover the correct Förster limit for large reorganization energies. This is a consequence of the choice of the electronic wavefunction (see eq. (37)), which is fixed and cannot adapt to the reorganization of vibrational degrees of freedom. ${ }^{128}$ Namely, when the reorganization energy is much larger than the coupling, electronic states should be completely localized, but in the Redfield theories the degree of localization is independent of the SD.

From the above discussion, it is clear that $\mathrm{mR}$ theory can describe an intermediate regime 
between Förster and standard Redfield only when the coupling to vibrations is sufficiently small. In this case, the main source of localization is static disorder in the energies of the chromophores, and the electronic wavefunctions are weakly perturbed by the vibrations. In the presence of static disorder, the average energy gap between chromophores is roughly proportional to the width of the energy distribution, $\sigma$. Therefore, for $\mathrm{mR}$ theory to describe correct dynamics, one has to ensure that either $V \gg \lambda$ or $\sigma \sim \lambda \gtrsim V$. In the other cases, EET is likely better modeled by Förster theory, assuming complete localization of the excitation, or by GF theory, assuming partial localization of the excitation on pre-defined clusters.

\section{Overcoming perturbative treatments}

All the methods described above assume that the electronic wavefunctions do not change upon relaxation of the nuclear coordinates, remaining either delocalized, as assumed in Redfield approaches, localized on one chromophore (Förster theory), or on a cluster of chromophores, as in GF theory. In some cases, however, the excitation can gradually localize while the nuclear degrees of freedom relax. This phenomenon is known as dynamic localization, and cannot be described by the perturbative treatments described in the previous sections. ${ }^{74,128,135}$ A number of methods have been devised to treat the quantum dynamics of energy transfer beyond the perturbative theories. ${ }^{39}$ The exact exciton dynamics of an exciton system can be described through a series of hierarchically coupled equations of motion (HEOM). The HEOM approach was derived in a reduced form by Ishizaki and Fleming, ${ }^{132}$ and is now widely used to consistently describe all ranges of exciton-phonon couplings ${ }^{121,135-137}$ and to benchmark the simpler theories. ${ }^{132,138}$

From the comparison with HEOM dynamics it was shown that Redfield-based theories easily give qualitatively incorrect dynamics outside of their range of applicability. ${ }^{132,135,138,139}$ Moreover, Redfield theories incorrectly predict, in the long-time limit, that the populations will equilibrate to the Boltzmann population of exciton states, regardless of the reorganization energies. ${ }^{136}$ The real equilibrium populations, however, will reflect the mixing with nuclear degrees of freedom, which effectively renormalizes the eigenstates of the full Hamiltonian. ${ }^{136,140}$ Förster and generalized Förster theory describe qualitatively well the EET rates even when the electronic couplings are not very small, and predict qualitatively correct 
populations at equilibrium. ${ }^{121,132,138,141}$

To overcome the limitations of the HEOM due to numerical complexity, several implementations have been devised, including massively parallel implementations, ${ }^{137,141}$ or stochastic approaches. ${ }^{142}$ However, other limitations remain, on the functional form of the spectral density, and on the number of vibrational modes that can be modeled. ${ }^{141}$

\section{CONCLUSIONS}

Nowadays, simulations of EET processes have achieved a considerable degree of maturity. In this review, we provided an overview of the main theoretical aspects involved in such simulations focusing mostly on biological macromolecules. We started by describing the main approximations in the original formulation of Förster theory. We then presented the improvements that have been devised to better estimate the electronic coupling, which is the key quantity determining the EET rate. In particular, methods that go beyond the pointdipole approximation have been presented and discussed together with models developed to properly include environment effects. We thus focused on recent advances in continuum and multiscale QM/MM methods, which show that screening effects can be significantly modulated by the chromophores mutual arrangement as well as the heterogeneous polarizability of the environment. Indeed, application of QM/MM models to DNA or photosynthetic antennae has shown changes by a factor up to 4 in energy transfer rates compared to the continuum dielectric assumption. We have also shown that, especially in biomacromolecules, the interplay between timescales of the EET process and the fluctuations of the coupling, the latter caused by chromophore rotational and translational dynamics, is fundamental to determine EET efficiencies. We thus discussed different strategies to post-process coupling trajectories and generate EET observables in the static, dynamic and intermediate averaging regimes. We successively analyzed the spectral overlap term and how this quantity can be estimated from simulations of the spectral density of exciton-phonon coupling. Finally, we ended by discussing rate theories beyond Förster formulation that need to be used in closely packed multichromophoric systems, where strong coupling leads to delocalized exciton states, a common situation found in biosystems like nucleic acids or photosynthetic complexes. We 
envision that such simulations will play an increasingly important role in the emerging field of dynamic structural biology, for example in the determination of conformational ensembles of intrinsically-disordered proteins, as well as in the comprehension of fundamental biological process like light harvesting in photosynthesis and the mechanisms of DNA damage.

\section{ACKNOWLEDGEMENTS}

Financial support from the Agència de Gestió d'Ajuts Universitaris i de Recerca from Generalitat de Catalunya (GENCAT; SGR2014-1189 and SGR2017-1746) and from the Spanish Agencia Estatal de Investigación and the European Regional Development Fund (AEI/FEDER/UE; grant CTQ2017-89924-P) are acknowledged. C.C. is a Serra Húnter Fellow (Generalitat de Catalunya) and M.C. acknowledges a fellowship from the Spanish Ministerio de Economía y Competitividad (MINECO; grant BES-2013-064088).

\section{References}

[1] Scholes GD. Long-range resonance energy transfer in molecular systems. Annu Rev Phys Chem. 2003;54:57-87.

[2] Förster T. Zwischenmolekulare Energiewanderung und Fluoreszenz. Ann Phys. 1948;437:55-75.

[3] Kramer HEA, Fischer P. The Scientific Work of Theodor Förster: A Brief Sketch of his Life and Personality. ChemPhysChem. 2011;12:555-558.

[4] Medintz I, Hildebrandt N, editors. FRET - Förster Resonance Energy Transfer. Weinheim, Germany: Wiley-VCH Verlag GmbH \& Co. KGaA; 2014.

[5] Lerner E, Cordes T, Ingargiola A, Alhadid Y, Chung S, Michalet X, et al. Toward dynamic structural biology: Two decades of single-molecule Förster resonance energy transfer. Science. 2018;359:eaan1133. 
[6] Schuler B, Eaton WA. Protein folding studied by single-molecule FRET. Curr Opin Struct Biol. 2008;18:16-26.

[7] Mirkovic T, Ostroumov EE, Anna JM, van Grondelle R, Govindjee, Scholes GD. Light Absorption and Energy Transfer in the Antenna Complexes of Photosynthetic Organisms. Chem Rev. 2017;117:249-293.

[8] Curutchet C, Mennucci B. Quantum Chemical Studies of Light Harvesting. Chem Rev. 2017;117:294-343.

[9] Voityuk AA, Marcus RA, Michel-Beyerle ME. On the mechanism of photoinduced dimer dissociation in the plant UVR8 photoreceptor. Proc Natl Acad Sci. $2014 ; 111: 5219-5224$.

[10] Markovitsi D, Gustavsson T, Vayá I. Fluorescence of DNA Duplexes: From Model Helices to Natural DNA. J Phys Chem Lett. 2010;1:3271-3276.

[11] Beljonne D, Curutchet C, Scholes GD, Silbey RJ. Beyond Forster Resonance Energy Transfer in Biological and Nanoscale Systems. J Phys Chem B. 2009;113:6583-6599.

[12] Braslavsky SE, Fron E, Rodríguez HB, Román ES, Scholes GD, Schweitzer G, et al. Pitfalls and limitations in the practical use of Förster's theory of resonance energy transfer. Photochem Photobiol Sci. 2008;7:1444.

[13] Schuler B, Lipman EA, Steinbach PJ, Kumke M, Eaton WA. Polyproline and the "spectroscopic ruler" revisited with single-molecule fluorescence. Proc Natl Acad Sci. $2005 ; 102: 2754-2759$.

[14] Jean JM, Krueger BP. Structural fluctuations and excitation transfer between adenine and 2-aminopurine in single-stranded deoxytrinucleotides. J Phys Chem B. 2006;110:2899-909.

[15] Beierlein FR, Othersen OG, Lanig H, Schneider S, Clark T. Simulating FRET from Tryptophan: Is the Rotamer Model Correct? J Am Chem Soc. 2006;128:5142-5152. 
[16] VanBeek DB, Zwier MC, Shorb JM, Krueger BP. Fretting about FRET: Correlation between $\kappa$ and R. Biophys J. 2007;92:4168-4178.

[17] Merchant KA, Best RB, Louis JM, Gopich IV, Eaton WA. Characterizing the unfolded states of proteins using single-molecule FRET spectroscopy and molecular simulations. Proc Natl Acad Sci. 2007;104:1528-1533.

[18] Dolghih E, Roitberg AE, Krause JL. Fluorescence resonance energy transfer in dyelabeled DNA. J Photochem Photobiol A Chem. 2007;190:321-327.

[19] Wozniak AK, Schroder GF, Grubmuller H, Seidel CAM, Oesterhelt F. Single-molecule FRET measures bends and kinks in DNA. Proc Natl Acad Sci. 2008;105:18337-18342.

[20] Allen LR, Paci E. Orientational averaging of dye molecules attached to proteins in Forster resonance energy transfer measurements: Insights from a simulation study. J Chem Phys. 2009;131:065101.

[21] Dolghih E, Ortiz W, Kim S, Krueger BP, Krause JL, Roitberg AE. Theoretical Studies of Short Polyproline Systems: Recalibration of a Molecular Ruler . J Phys Chem A. 2009;113:4639-4646.

[22] Unruh JR, Kuczera K, Johnson CK. Conformational Heterogeneity of a Leucine Enkephalin Analogue in Aqueous Solution and Sodium Dodecyl Sulfate Micelles: Comparison of Time-Resolved FRET and Molecular Dynamics Simulations. J Phys Chem B. 2009;113:14381-14392.

[23] Stevens JA, Link JJ, Kao YT, Zang C, Wang L, Zhong D. Ultrafast Dynamics of Resonance Energy Transfer in Myoglobin: Probing Local Conformation Fluctuations. J Phys Chem B. 2010;114:1498-1505.

[24] Speelman AL, Munoz-Losa A, Hinkle KL, VanBeek DB, Mennucci B, Krueger BP. Using Molecular Dynamics and Quantum Mechanics Calculations To Model Fluorescence Observables. J Phys Chem A. 2011;115:3997-4008. 
[25] Hoefling M, Lima N, Haenni D, Seidel CAM, Schuler B, Grubmüller H. Structural Heterogeneity and Quantitative FRET Efficiency Distributions of Polyprolines through a Hybrid Atomistic Simulation and Monte Carlo Approach. PLoS One. 2011;6:e19791.

[26] Kalinin S, Peulen T, Sindbert S, Rothwell PJ, Berger S, Restle T, et al. A toolkit and benchmark study for FRET-restrained high-precision structural modeling. Nat Methods. 2012;9:1218-1225.

[27] Stevens JA, Link JJ, Zang C, Wang L, Zhong D. Ultrafast Dynamics of Nonequilibrium Resonance Energy Transfer and Probing Globular Protein Flexibility of Myoglobin. J Phys Chem A. 2012;116:2610-2619.

[28] Milas P, Gamari BD, Parrot L, Krueger BP, Rahmanseresht S, Moore J, et al. Indocyanine Dyes Approach Free Rotation at the 3 Terminus of A-RNA: A Comparison with the 5 Terminus and Consequences for Fluorescence Resonance Energy Transfer. J Phys Chem B. 2013;117:8649-8658.

[29] Hoefling M, Grubmüller H. In silico FRET from simulated dye dynamics. Comput Phys Commun. 2013;184:841-852.

[30] Kellner R, Hofmann H, Barducci A, Wunderlich B, Nettels D, Schuler B. Singlemolecule spectroscopy reveals chaperone-mediated expansion of substrate protein. Proc Natl Acad Sci. 2014;111:13355-13360.

[31] Reif MM, Oostenbrink C. Molecular dynamics simulation of configurational ensembles compatible with experimental FRET efficiency data through a restraint on instantaneous FRET efficiencies. J Comput Chem. 2014;35:2319-2332.

[32] Shoura MJ, Ranatunga RJKU, Harris SA, Nielsen SO, Levene SD. Contribution of Fluorophore Dynamics and Solvation to Resonant Energy Transfer in Protein-DNA Complexes: A Molecular-Dynamics Study. Biophys J. 2014;107:700-710.

[33] Søndergaard S, Aznauryan M, Haustrup EK, Schiøtt B, Birkedal V, Corry B. Dynamics of Fluorescent Dyes Attached to G-Quadruplex DNA and their Effect on FRET Experiments. ChemPhysChem. 2015;16:2562-2570. 
[34] Kulesza A, Daly S, MacAleese L, Antoine R, Dugourd P. Structural exploration and Förster theory modeling for the interpretation of gas-phase FRET measurements: Chromophore-grafted amyloid- $\beta$ peptides. J Chem Phys. 2015;143:025101.

[35] Spiegel JD, Fulle S, Kleinschmidt M, Gohlke H, Marian CM. Failure of the IDA in FRET Systems at Close Inter-Dye Distances Is Moderated by Frequent Low $\kappa 2$ Values. J Phys Chem B. 2016;120:8845-8862.

[36] Kitoh-Nishioka H, Yokogawa D, Irle S. Förster Resonance Energy Transfer between Fluorescent Proteins: Efficient Transition Charge-Based Study. J Phys Chem C. 2017;121:4220-4238.

[37] Meng F, Bellaiche MMJ, Kim JY, Zerze GH, Best RB, Chung HS. Highly Disordered Amyloid- $\beta$ Monomer Probed by Single-Molecule FRET and MD Simulation. Biophys J. 2018;114:870-884.

[38] Sobakinskaya E, Schmidt am Busch M, Renger T. Theory of FRET Spectroscopic Ruler for Short Distances: Application to Polyproline. J Phys Chem B. 2018;122:54-67.

[39] Chenu A, Scholes GD. Coherence in Energy Transfer and Photosynthesis. Annu Rev Phys Chem. 2015;66:69-96.

[40] You ZQ, Hsu CP. Theory and calculation for the electronic coupling in excitation energy transfer. Int J Quantum Chem. 2014;114:102-115.

[41] Dexter DL. A Theory of Sensitized Luminescence in Solids. J Chem Phys. 1953;21:836.

[42] Scholes GD, Fleming GR. 2. In: Energy Transfer and Photosynthetic Light Harvesting. Wiley-Blackwell; 2005. p. 57-129.

[43] Hsu CP. The electronic couplings in electron transfer and excitation energy transfer. Acc Chem Res. 2009;42:509-18.

[44] Hsu CP, Fleming GR, Head-Gordon M, Head-Gordon T. Excitation energy transfer in condensed media. J Chem Phys. 2001;114:3065. 
[45] Voityuk AA. Interaction of Dark Excited States. Comparison of Computational Approaches. J Phys Chem B. 2015;119:7417-7421.

[46] Muñoz-Losa A, Curutchet C, Krueger BP, Hartsell LR, Mennucci B. Fretting about FRET: Failure of the Ideal Dipole Approximation. Biophys J. 2009;96:4779-4788.

[47] Iozzi MF, Mennucci B, Tomasi J, Cammi R. Excitation energy transfer (EET) between molecules in condensed matter: A novel application of the polarizable continuum model (PCM). J Chem Phys. 2004;120:7029-7040.

[48] Damjanović A, Ritz T, Schulten K. Energy transfer between carotenoids and bacteriochlorophylls in light-harvesting complex II of purple bacteria. Phys Rev E. 1999;59:3293-3311.

[49] Damjanović A, Ritz T, Schulten K. Excitation transfer in the peridinin-chlorophyllprotein of Amphidinium carterae. Biophys J. 2000;79:1695-705.

[50] Nagae H, Kakitani T, Katoh T, Mimuro M. Calculation of the excitation transfer matrix elements between the S2 or S1 state of carotenoid and the S2 or S1 state of bacteriochlorophyll. J Chem Phys. 1993;98:8012.

[51] Wong KF, Bagchi B, Rossky PJ. Distance and Orientation Dependence of Excitation Transfer Rates in Conjugated Systems: Beyond the Förster Theory. J Phys Chem A. 2004;108:5752-5763.

[52] Krueger BP, Scholes GD, Fleming GR. Calculation of Couplings and Energy-Transfer Pathways between the Pigments of LH2 by the ab Initio Transition Density Cube Method. J Phys Chem B. 1998;102:5378-5386.

[53] Hsu CP, Walla PJ, Head-Gordon M, Fleming GR. The Role of the S 1 State of Carotenoids in Photosynthetic Energy Transfer: The Light-Harvesting Complex II of Purple Bacteria. J Phys Chem B. 2001;105:11016-11025.

[54] Muñoz-Losa A, Curutchet C, Galván IF, Mennucci B. Quantum mechanical methods applied to excitation energy transfer: A comparative analysis on excitation energies and electronic couplings. J Chem Phys. 2008;129:034104. 
[55] Caricato M, Curutchet C, Mennucci B, Scalmani G. Electronic Couplings for Resonance Energy Transfer from CCSD Calculations: From Isolated to Solvated Systems. J Chem Theory Comput. 2015;11:5219-5228.

[56] Fückel B, Köhn A, Harding ME, Diezemann G, Hinze G, Basché T, et al. Theoretical investigation of electronic excitation energy transfer in bichromophoric assemblies. J Chem Phys. 2008;128:074505.

[57] Kistler KA, Spano FC, Matsika S. A Benchmark of Excitonic Couplings Derived from Atomic Transition Charges. J Phys Chem B. 2013;117:2032-2044.

[58] Howard IA, Zutterman F, Deroover G, Lamoen D, Van Alsenoy C. Approaches to Calculation of Exciton Interaction Energies for a Molecular Dimer. J Phys Chem B. 2004;108:19155-19162.

[59] Duffy CDP, Chmeliov J, Macernis M, Sulskus J, Valkunas L, Ruban aV. Modeling of Fluorescence Quenching by Lutein in the Plant Light-Harvesting Complex LHCII. J Phys Chem B. 2013;117:10974-10986.

[60] Madjet ME, Abdurahman A, Renger T. Intermolecular coulomb couplings from ab initio electrostatic potentials: Application to optical transitions of strongly coupled pigments in photosynthetic antennae and reaction centers. J Phys Chem B. 2006;110:17268-17281.

[61] Olbrich C, Jansen TLC, Liebers J, Aghtar M, Strumpfer J, Schulten K, et al. From Atomistic Modeling to Excitation Transfer and Two-Dimensional Spectra of the FMO Light-Harvesting Complex. J Phys Chem B. 2011;115:8609-8621.

[62] van der Vegte CP, Prajapati JD, Kleinekathöfer U, Knoester J, Jansen TLC. Atomistic Modeling of Two-Dimensional Electronic Spectra and Excited-State Dynamics for a Light Harvesting 2 Complex. J Phys Chem B. 2015;119:1302-1313.

[63] Olbrich C, Kleinekathöfer U. Time-dependent atomistic view on the electronic relaxation in light-harvesting system II. J Phys Chem B. 2010;114:12427-37. 
[64] Maj M, Jeon J, Góra RW, Cho M. Induced Optical Activity of DNA-Templated Cyanine Dye Aggregates: Exciton Coupling Theory and TD-DFT Studies. J Phys Chem A. 2013;117:5909-5918.

[65] Fujimoto KJ. Electronic coupling calculations with transition charges, dipoles, and quadrupoles derived from electrostatic potential fitting. J Chem Phys. 2014;141:214105.

[66] Błasiak B, Maj M, Cho M, Góra RW. Distributed Multipolar Expansion Approach to Calculation of Excitation Energy Transfer Couplings. J Chem Theory Comput. 2015;11:3259-3266.

[67] Knox RS, Amerongen HV. Refractive Index Dependence of the Foerster Resonance Excitation Transfer Rate. J Phys Chem B. 2002;106:5289-5293.

[68] Tomasi J, Mennucci B, Cammi R. Quantum mechanical continuum solvation models. Chem Rev. 2005;105:2999-3093.

[69] Adolphs J, Renger T. How Proteins Trigger Excitation Energy Transfer in the FMO Complex of Green Sulfur Bacteria. Biophys J. 2006;91:2778-2797.

[70] Renger T, Müh F. Theory of excitonic couplings in dielectric media. Photosynth Res. $2012 ; 111: 47-52$.

[71] Scholes GD, Curutchet C, Mennucci B, Cammi R, Tomasi J. How Solvent Controls Electronic Energy Transfer and Light Harvesting. J Phys Chem B. 2007;111:6978-6982.

[72] Curutchet C, Scholes GD, Mennucci B, Cammi R. How solvent controls electronic energy transfer and light harvesting: toward a quantum-mechanical description of reaction field and screening effects. J Phys Chem B. 2007;111:13253-65.

[73] Megow J, Renger T, May V. Mixed Quantum-Classical Description of Excitation Energy Transfer in Supramolecular Complexes: Screening of the Excitonic Coupling. ChemPhysChem. 2014;15:478-485. 
[74] Renger T, Müh F. Understanding photosynthetic light-harvesting: a bottom up theoretical approach. Phys Chem Chem Phys. 2013;15:3348.

[75] Curutchet C, Munoz-Losa A, Monti S, Kongsted J, Scholes GD, Mennucci B. Electronic Energy Transfer in Condensed Phase Studied by a Polarizable QM/MM Model. J Chem Theory Comput. 2009;5:1838-1848.

[76] Curutchet C, Kongsted J, Muñoz-Losa A, Hossein-Nejad H, Scholes GD, Mennucci B. Photosynthetic light-harvesting is tuned by the heterogeneous polarizable environment of the protein. J Am Chem Soc. 2011;133:3078-84.

[77] Steinmann C, Kongsted J. Electronic Energy Transfer in Polarizable Heterogeneous Environments: A Systematic Investigation of Different Quantum Chemical Approaches. J Chem Theory Comput. 2015;11:4283-4293.

[78] Jurinovich S, Curutchet C, Mennucci B. The Fenna-Matthews-Olson protein revisited: a fully polarizable (TD)DFT/MM description. Chemphyschem. 2014;15:3194-204.

[79] Jurinovich S, Viani L, Prandi IG, Renger T, Mennucci B. Towards an ab initio description of the optical spectra of light-harvesting antennae: application to the CP29 complex of photosystem II. Phys Chem Chem Phys. 2015;17:14405-16.

[80] Cupellini L, Jurinovich S, Campetella M, Caprasecca S, Guido CA, Kelly SM, et al. An Ab Initio Description of the Excitonic Properties of LH2 and Their Temperature Dependence. J Phys Chem B. 2016;120:11348-11359.

[81] Loco D, Jurinovich S, Di Bari L, Mennucci B. A fast but accurate excitonic simulation of the electronic circular dichroism of nucleic acids: how can it be achieved? Phys Chem Chem Phys. 2016;18:866-877.

[82] Padula D, Jurinovich S, Di Bari L, Mennucci B. Simulation of Electronic Circular Dichroism of Nucleic Acids: From the Structure to the Spectrum. Chem - A Eur J. 2016;22:17011-17019. 
[83] Pinheiro S, Curutchet C. Can Förster Theory Describe Stereoselective Energy Transfer Dynamics in a Protein-Ligand Complex? J Phys Chem B. 2017;121:2265-2278.

[84] Nottoli M, Jurinovich S, Cupellini L, Gardiner AT, Cogdell R, Mennucci B. The role of charge-transfer states in the spectral tuning of antenna complexes of purple bacteria. Photosynth Res. 2018;137:215-226.

[85] Neugebauer J, Curutchet C, Munoz-Losa A, Mennucci B. A Subsystem TDDFT Approach for Solvent Screening Effects on Excitation Energy Transfer Couplings. J Chem Theory Comput. 2010;6:1843-1851.

[86] Neugebauer J. Couplings between electronic transitions in a subsystem formulation of time-dependent density functional theory. J Chem Phys. 2007;126:134116.

[87] Subotnik JE, Alguire EC, Ou Q, Landry BR, Fatehi S. The Requisite Electronic Structure Theory To Describe Photoexcited Nonadiabatic Dynamics: Nonadiabatic Derivative Couplings and Diabatic Electronic Couplings. Acc Chem Res. 2015;48:13401350.

[88] Hoyer CE, Xu X, Ma D, Gagliardi L, Truhlar DG. Diabatization based on the dipole and quadrupole: The DQ method. J Chem Phys. 2014;141:114104.

[89] Aragó J, Troisi A. Excitonic couplings between molecular crystal pairs by a multistate approximation. J Chem Phys. 2015;142.

[90] Fornari RP, Rowe P, Padula D, Troisi A. Importance and Nature of Short-Range Excitonic Interactions in Light Harvesting Complexes and Organic Semiconductors. J Chem Theory Comput. 2017;13:3754-3763.

[91] Yeganeh S, Voorhis TV. Triplet Excitation Energy Transfer with Constrained Density Functional Theory. J Phys Chem C. 2010;114:20756-20763.

[92] Hsu CP, You ZQ, Chen HC. Characterization of the Short-Range Couplings in Excitation Energy Transfer. J Phys Chem C. 2008;112:1204-1212. 
[93] Voityuk AA, Rösch N. Fragment charge difference method for estimating donoracceptor electronic coupling: Application to DNA $\pi$-stacks. J Chem Phys. 2002;117:5607.

[94] You ZQ, Hsu CP. The fragment spin difference scheme for triplet-triplet energy transfer coupling. J Chem Phys. 2010;133:074105.

[95] Voityuk AA. Fragment transition density method to calculate electronic coupling for excitation energy transfer. J Chem Phys. 2014;140:244117.

[96] Ritze HH, Hobza P, Nachtigallová D. Electronic coupling in the excited electronic state of stacked DNA base homodimers. Phys Chem Chem Phys. 2007;9:1672.

[97] Fink RF, Pfister J, Zhao HM, Engels B. Assessment of quantum chemical methods and basis sets for excitation energy transfer. Chem Phys. 2008;346:275-285.

[98] Curutchet C, Voityuk AA. Triplet-Triplet Energy Transfer in DNA: A Process that Occurs on the Nanosecond Timescale. Angew Chemie. 2011;123:1860-1862.

[99] Blancafort L, Voityuk AA. Thermally induced hopping model for long-range triplet excitation energy transfer in DNA. Phys Chem Chem Phys. 2018;20:4997-5000.

[100] Si Y, Yang B, Qin H, Yuan J, Wang S, Chen H, et al. Atomistic Modeling of TripletTriplet Energy-Transfer Rates from Drug (S)-Propranolol to (R)-Cinacalcet in Human $\alpha 1$-Acid Glycoprotein. J Phys Chem C. 2015;119:8014-8022.

[101] Cupellini L, Jurinovich S, Prandi IG, Caprasecca S, Mennucci B. Photoprotection and triplet energy transfer in higher plants: the role of electronic and nuclear fluctuations. Phys Chem Chem Phys. 2016;18:11288-11296.

[102] Renger T, Madjet MEA, Schmidt am Busch M, Adolphs J, Müh F. Structure-based modeling of energy transfer in photosynthesis. Photosynth Res. 2013;116:367-388.

[103] König C, Neugebauer J. Quantum Chemical Description of Absorption Properties and Excited-State Processes in Photosynthetic Systems. ChemPhysChem. 2012;13:386425. 
[104] Aghtar M, Kleinekathöfer U, Curutchet C, Mennucci B. Impact of Electronic Fluctuations and Their Description on the Exciton Dynamics in the Light-Harvesting Complex PE545. J Phys Chem B. 2017;121:1330-1339.

[105] You ZQ, Hsu CP. Ab inito study on triplet excitation energy transfer in photosynthetic light-harvesting complexes. J Phys Chem A. 2011;115:4092-100.

[106] Scholes GD, Fleming GR. On the Mechanism of Light Harvesting in Photosynthetic Purple Bacteria: B800 to B850 Energy Transfer. J Phys Chem B. 2000;104:1854-1868.

[107] Mukamel S. Principles of Nonlinear Optical Spectroscopy. New York: Oxford University Press; 1995.

[108] Valleau S, Eisfeld A, Aspuru-Guzik A. On the alternatives for bath correlators and spectral densities from mixed quantum-classical simulations. J Chem Phys. 2012;137:224103.

[109] Yang M, Fleming GR. Third-order nonlinear optical response and energy transfer in static disordered systems. J Chem Phys. 2000;113:2823.

[110] Yang M, Fleming GR. Influence of phonons on exciton transfer dynamics: comparison of the Redfield, Förster, and modified Redfield equations. Chem Phys. 2002;275:355372.

[111] Hennebicq E, Beljonne D, Curutchet C, Scholes GD, Silbey RJ. Shared-mode assisted resonant energy transfer in the weak coupling regime. J Chem Phys. 2009;130:214505.

[112] Chandrasekaran S, Aghtar M, Valleau S, Aspuru-Guzik A, Kleinekathöfer U. Influence of Force Fields and Quantum Chemistry Approach on Spectral Densities of BChl a in Solution and in FMO Proteins. J Phys Chem B. 2015;119:9995-10004.

[113] Shim S, Rebentrost P, Valleau S, Aspuru-Guzik A. Atomistic Study of the LongLived Quantum Coherences in the Fenna-Matthews-Olson Complex. Biophys J. 2012;102:649-660. 
[114] Lee MK, Huo P, Coker DF. Semiclassical Path Integral Dynamics: Photosynthetic Energy Transfer with Realistic Environment Interactions. Annu Rev Phys Chem. 2016;67:639-668.

[115] Lee MK, Coker DF. Modeling Electronic-Nuclear Interactions for Excitation Energy Transfer Processes in Light-Harvesting Complexes. J Phys Chem Lett. 2016;7:31713178.

[116] Zwier MC, Shorb JM, Krueger BP. Hybrid molecular dynamics-quantum mechanics simulations of solute spectral properties in the condensed phase: evaluation of simulation parameters. J Comput Chem. 2007;28:1572-81.

[117] Kim CW, Park JW, Rhee YM. Effect of Chromophore Potential Model on the Description of Exciton-Phonon Interactions. J Phys Chem Lett. 2015;6:2875-2880.

[118] Aghtar M, Strümpfer J, Olbrich C, Schulten K, Kleinekathöfer U. The FMO complex in a glycerol-water mixture. J Phys Chem B. 2013;117:7157-63.

[119] Loco D, Jurinovich S, Cupellini L, Menger MFSJ, Mennucci B. The modeling of the absorption lineshape for embedded molecules through a polarizable QM/MM approach. Photochem Photobiol Sci. 2018;17:552-560.

[120] Rosnik AM, Curutchet C. Theoretical Characterization of the Spectral Density of the Water-Soluble Chlorophyll-Binding Protein from Combined Quantum Mechanics/Molecular Mechanics Molecular Dynamics Simulations. J Chem Theory Comput. 2015; $11: 5826-5837$.

[121] Blau SM, Bennett DIG, Kreisbeck C, Scholes GD, Aspuru-Guzik A. Local protein solvation drives direct down-conversion in phycobiliprotein PC645 via incoherent vibronic transport. Proc Natl Acad Sci. 2018;115:E3342-E3350.

[122] Prandi IG, Viani L, Andreussi O, Mennucci B. Combining classical molecular dynamics and quantum mechanical methods for the description of electronic excitations: The case of carotenoids. J Comput Chem. 2016;37:981-991. 
[123] Andreussi O, Prandi IG, Campetella M, Prampolini G, Mennucci B. Classical Force Fields Tailored for QM Applications: Is It Really a Feasible Strategy? J Chem Theory Comput. 2017;13:4636-4648.

[124] Kim CW, Choi B, Rhee YM. Excited state energy fluctuations in the Fenna-MatthewsOlson complex from molecular dynamics simulations with interpolated chromophore potentials. Phys Chem Chem Phys. 2018;20:3310-3319.

[125] Lee MK, Bravaya KB, Coker DF. First-Principles Models for Biological LightHarvesting: Phycobiliprotein Complexes from Cryptophyte Algae. J Am Chem Soc. 2017;139:7803-7814.

[126] Segatta F, Cupellini L, Jurinovich S, Mukamel S, Dapor M, Taioli S, et al. A Quantum Chemical Interpretation of Two-Dimensional Electronic Spectroscopy of LightHarvesting Complexes. J Am Chem Soc. 2017;139:7558-7567.

[127] Cheng YC, Fleming GR. Dynamics of Light Harvesting in Photosynthesis. Annu Rev Phys Chem. 2009;60:241-262.

[128] Novoderezhkin VI, van Grondelle R. Physical origins and models of energy transfer in photosynthetic light-harvesting. Phys Chem Chem Phys. 2010;12:7352-65.

[129] Sumi H. Theory on Rates of Excitation-Energy Transfer between Molecular Aggregates through Distributed Transition Dipoles with Application to the Antenna System in Bacterial Photosynthesis. J Phys Chem B. 1999;103:252-260.

[130] Jang S, Newton MD, Silbey RJ. Multichromophoric Förster resonance energy transfer. Phys Rev Lett. 2004;92:21-24.

[131] Ma J, Moix J, Cao J. Förster resonance energy transfer, absorption and emission spectra in multichromophoric systems. II. Hybrid cumulant expansion. J Chem Phys. $2015 ; 142$.

[132] Ishizaki A, Fleming GR. Unified treatment of quantum coherent and incoherent hopping dynamics in electronic energy transfer: Reduced hierarchy equation approach. J Chem Phys. 2009;130:234111. 
[133] Zhang WM, Meier T, Chernyak V, Mukamel S. Exciton-migration and three-pulse femtosecond optical spectroscopies of photosynthetic antenna complexes. J Chem Phys. 1998;108:7763.

[134] Renger T, Marcus RA. On the relation of protein dynamics and exciton relaxation in pigment-protein complexes: An estimation of the spectral density and a theory for the calculation of optical spectra. J Chem Phys. 2002;116:9997-10019.

[135] Fassioli F, Dinshaw R, Arpin PC, Scholes GD. Photosynthetic light harvesting: excitons and coherence. J R Soc Interface. 2013;11:20130901-20130901.

[136] Balevičius V, Gelzinis A, Abramavicius D, Mančal T, Valkunas L. Excitation dynamics and relaxation in a molecular heterodimer. Chem Phys. 2012;404:94-102.

[137] Kramer T, Noack M, Reimers JR, Reinefeld A, Rodríguez M, Yin S. Energy flow in the Photosystem I supercomplex: Comparison of approximative theories with DM-HEOM. Chem Phys. 2018;(DOI: 10.1016/j.chemphys.2018.05.028).

[138] Novoderezhkin VI, van Grondelle R. Spectra and Dynamics in the B800 Antenna: Comparing Hierarchical Equations, Redfield and Förster Theories. J Phys Chem B. 2013;117:11076-11090.

[139] Ishizaki A, Fleming GR. On the adequacy of the Redfield equation and related approaches to the study of quantum dynamics in electronic energy transfer. J Chem Phys. 2009;130:234110.

[140] Gelzinis A, Abramavicius D, Valkunas L. Non-Markovian effects in time-resolved fluorescence spectrum of molecular aggregates: Tracing polaron formation. Phys Rev B - Condens Matter Mater Phys. 2011;84:245430.

[141] Kreisbeck C, Kramer T, Aspuru-Guzik A. Scalable High-Performance Algorithm for the Simulation of Exciton Dynamics. Application to the Light-Harvesting Complex II in the Presence of Resonant Vibrational Modes. J Chem Theory Comput. 2014;10:4045-4054. 
[142] Moix JM, Cao J. A hybrid stochastic hierarchy equations of motion approach to treat the low temperature dynamics of non-Markovian open quantum systems. J Chem Phys. 2013;139:134106. 